\title{
FIFTY YEARS OF THE FINITE NONPERIODIC TODA LATTICE: A GEOMETRIC AND TOPOLOGICAL VIEWPOINT
}

\author{
YUJI KODAMA ${ }^{1}$ AND BARBARA A. SHIPMAN ${ }^{2}$
}

\begin{abstract}
In 1967, Japanese physicist Morikazu Toda published a pair of seminal papers in the Journal of the Physical Society of Japan that exhibited soliton solutions to a chain of particles with nonlinear interactions between nearest neighbors. In the fifty years that followed, Toda's system of particles has been generalized in different directions, each with its own analytic, geometric, and topological characteristics. These are known collectively as the Toda lattice. This survey recounts and compares the various versions of the finite nonperiodic Toda lattice from the perspective of their geometry and topology. In particular, we highlight the polytope structure of the solution spaces as viewed through the moment map, and we explain the connection between the real indefinite Toda flows and the integral cohomology of real flag varieties.
\end{abstract}

\section{Contents}

1. Historical overview

2. Early versions of the finite nonperiodic real Toda lattice

2.1. Symmetric form

2.2. Hessenberg form

2.3. Isospectral manifolds in the real tridiagonal symmetric form

2.4. Indefinite Toda lattice in real tridiagonal Hessenberg form

2.5. Full symmetric real Toda lattice

3. Complex Toda lattices

3.1. The moment map

3.2. Complex tridiagonal Hessenberg form

3.3. The full Kostant-Toda lattice

3.4. Nongeneric full Kostant-Toda flows

4. Other Extensions of the Toda Lattice

4.1. The Kac-van Moerbeke system

4.2. The Pfaff lattice for a symplectic matrix

5. The full Kostant-Toda lattice in real variables

5.1. Totally nonnegative parts of flag varieties

5.2. Full Kostant-Toda flows with totally nonnegative initial data

5.3. Asymptotic behavior of the full Kostant-Toda lattice

5.4. The moment polytope of the full Kostant-Toda lattice

6. The Toda lattice and integral cohomology of real flag varieties

6.1. Integral cohomology of $G / B$

6.2. Blow-ups of the indefinite Toda lattice on $G$ and the cohomology of $G / B$

References

${ }^{1}$ Partially supported by NSF grants DMS-1410267 and DMS-1714770. 


\section{Historical OVERView}

In 1974, Henon 35] and Flaschka 24] announced the complete integrability of the (real, finite, periodic) Toda lattice. This came seven years after the pivotal papers of M. Toda on vibrations in chains with nonlinear interactions [73] and waves in anharmonic lattices [74. Again in 1974, Flaschka [24, 25], and also Manakov [56, showed that the periodic Toda lattice can be written in Lax form through a change of variables, so that the constants of motion appear as eigenvalues of the Lax matrix [53.

Six years later, Moser [59] showed that the (real, finite) nonperiodic Toda lattice is completely integrable. In two different expressions of the equations, the flows obey a Lax equation on a set of real tridiagonal Lax matrices with positive subdiagonal entries. The matrices are symmetric in one formulation and Hessenberg in the other - these are two different expressions of the very same system. The flows exist for all time and preserve the spectrum of the initial Lax matrix.

However, when we allow the entries on the subdiagonal to take on any real values, the tridiagonal symmetric and Hessenberg forms create two genuinely different dynamical systems. In the symmetric case, the flows exist for all time and the isospectral manifolds are compact [75, while in the Hessenberg form, the flows can blow up in finite time and the isospectral manifolds are not compact (see e.g. [45, 46]). Shortly after this work, the flows on full symmetric real matrices in generic case were shown to be completely integrable 18 with the introduction of additional constants of motion.

Section 2 describes these versions of the real, finite, nonperiodic Toda lattices with a focus on their geometry and topology. This represents the work on real Toda lattices during roughly the first twenty years after the Toda lattice was discovered in 1967.

By the time the Toda lattice had been known for 25 years, studies on nonperiodic complex versions began to appear. These are the focus of Section 3 This phase brings in the new idea of compactifying the flows through embeddings into flag varieties. In a seminal paper, Ercolani, Flaschka, and Haine 21] describe the Toda system on complex tridiagonal matrices in Hessenberg form. The complex flows again blow up in finite (complex) time, but they differ from the real in that they no longer preserve "signs" of the subdiagonal entries. A theorem on matrix factorizations [52] is used to embed the isospectral sets into a flag variety. There, the flows enter lower-dimensional cells, called the Bruhat cells in the Bruhat decomposition of the flag variety, at the blow-up times, where the singularity at a blow-up time is characterized by the Bruhat cell [27, 4, 13.

Two years after 21, integrability was extended to the full Kostant-Toda lattice in [22, where the system evolves on complex Hessenberg matrices with arbitrary entries everywhere below the diagonal. On isospectral sets with distinct eigenvalues, the flows generate a diagonal torus action under the appropriate embedding into a flag variety. Similar embeddings, derived from the companion and Jordan matrices of the spectrum, are helpful in understanding nongeneric flows where eigenvalues coincide [69] and in describing the compactified complex isospectral sets [70]. Coincidence of eigenvalues is seen in splittings of moment polytopes, which allows for a description of monodromy around nongeneric isospectral sets in special cases [66, 68].

Section 4 discusses other extensions of the finite nonperiodic Toda lattices. The Toda flow in Lax form is introduced on an arbitrary diagonalizable matrix in [47] and is integrated by inverse scattering (or equivalently, by factorization). The tridiagonal Hessenberg and symmetric Toda lattices, which are defined on the Lie algebra of type $A$ (that is, $\mathfrak{s l}_{n}$ ), are extended to semisimple Lie algebras using the Lie algebra splittings from the Gauss (or LU-) and QR-factorizations, respectively [10, 11].

Related hierarchies are the Kac-van Moerbeke system, which can be considered as a square root of the Toda lattice [33, 37, and the Pfaff lattice, which evolves on symplectic matrices, and is connected to the indefinite Toda lattice [1, 43, 44].

Section 5 considers the full Kostant-Toda hierarchy in the real variables [50]. It classifies the regular solutions of the hierarchy in terms of the totally nonnegative parts of the flag variety $G / B$ 
where $G=S L(n, \mathbb{R})$ and $B$ is the set of upper-triangular matrices. Using the moment map, the full Kostant-Toda flows are defined on the appropriate weight space, and it is shown that the closure of each flow forms an interesting convex polytope, which we call a Bruhat interval polytope (see also [76]). This section begins with a brief review of the totally nonnegative flag variety [57, 49. The goal is to describe the topological structure of the regular solutions of the full Kostant-Toda lattice for the real split algebra $\mathfrak{s l}_{n}(\mathbb{R})$.

Section 6 describes how the singular structure of blow-ups in solutions of the indefinite Toda lattice contains information about the integral cohomology of real flag varieties [14, 15. We consider the moment polytope, the image of the moment map of the isospectral variety, for the real split semisimple Lie algebra of $\mathfrak{s l}_{n}(\mathbb{R})$. The vertices of the polytope are the orbit of the Weyl group action [11, 28. These vertices correspond to the fixed points of the Toda flows. Each edge of the polytope can be considered as an orbit of the $\mathfrak{s l}_{2}(\mathbb{R})$ Toda lattice (the smallest nontrivial lattice). An orbit may be regular (without blow-ups) or singular (with blow-ups). One can then define a graph whose vertices are the fixed points and where two fixed points are connected by an edge if and only if the $\mathfrak{s l}_{2}(\mathbb{R})$ flow between them is regular. This turns out to be the incidence graph that gives the integral cohomology of the real flag variety. The total number of blow-ups in the Toda flows is related to the polynomial associated with the rational cohomology of a certain compact subgroup [9, 14, 15.

\section{EARly Versions of the Finite nONPERIOdic REAL TOdA LATtice}

Consider $n$ particles, each with mass 1 , arranged along a line at positions $q_{1}, \ldots, q_{n}$. Between each pair of adjacent particles, there is a force whose magnitude depends exponentially on the distance between them. Letting $p_{k}$ denote the momentum of the $k$ th particle, and noting that $\frac{d}{d t} q_{k}=p_{k}$ since each mass is 1 , the total energy of the system is the Hamiltonian

$$
H=\frac{1}{2} \sum_{k=1}^{n} p_{k}^{2}+\sum_{k=1}^{n-1} e^{-\left(q_{k+1}-q_{k}\right)}
$$

The equations of motion

$$
\frac{d q_{k}}{d t}=\frac{\partial H}{\partial p_{k}} \quad \text { and } \quad \frac{d p_{k}}{d t}=-\frac{\partial H}{\partial q_{k}}
$$

give the system of equations for the finite nonperiodic Toda lattice,

$$
\begin{cases}\frac{d q_{k}}{d t}=p_{k}, & k=1, \ldots, n, \\ \frac{d p_{k}}{d t}=-e^{-\left(q_{k+1}-q_{k}\right)}+e^{-\left(q_{k}-q_{k-1}\right)}, & k=1, \ldots, n .\end{cases}
$$

Here we set $e^{-\left(q_{1}-q_{0}\right)}=0$ and $e^{-\left(q_{n+1}-q_{n}\right)}=0$ with the formal boundary conditions $q_{0}=-\infty$, and $q_{n+1}=\infty$.

2.1. Symmetric form. There are two classic Lax forms of Equations (2.3): the symmetric form and the Hessenberg form. For the symmetric form, we make the change of variables (Flaschka 24], Moser [59])

$$
\left\{\begin{aligned}
a_{k} & =\frac{1}{2} e^{-\frac{1}{2}\left(q_{k+1}-q_{k}\right)}, & k & =1, \ldots, n-1 \\
b_{k} & =-\frac{1}{2} p_{k}, & k & =1, \ldots, n .
\end{aligned}\right.
$$


In these variables, the Toda system (2.3) becomes

$$
\left\{\begin{aligned}
\frac{d a_{k}}{d t} & =a_{k}\left(b_{k+1}-b_{k}\right), & & k=1, \ldots, n-1 \\
\frac{d b_{k}}{d t} & =2\left(a_{k}^{2}-a_{k-1}^{2}\right), & k & =1, \ldots, n
\end{aligned}\right.
$$

with boundary conditions $a_{0}=0$ and $a_{n}=0$. Because the $a_{k}$ are real exponential functions, they are strictly positive for all time.

Remark 2.1. Making a change in sign $a_{k} \leftrightarrow-a_{k}$ for one or more values of $k$ in Definition 2.4 does not change Equations (2.5). That is, the systems $\left( \pm a_{k}, b_{k}\right)$ are equivalent for all choices of signs.

The system (2.5) can be written in Lax form as

$$
\frac{d}{d t} L(t)=\left[\Pi_{\mathfrak{s o}}(L(t)), L(t)\right]
$$

where $L$ is the symmetric tridiagonal matrix, and $\Pi_{\mathfrak{s o}}(L)$ is the skew-symmetric projection of $L$,

$$
L=\left(\begin{array}{cccc}
b_{1} & a_{1} & & \\
a_{1} & \ddots & \ddots & \\
& \ddots & \ddots & a_{n-1} \\
& & a_{n-1} & b_{n}
\end{array}\right) \quad \text { and } \quad \Pi_{\mathfrak{s o}}(L)=(L)_{>0}-(L)_{<0} .
$$

Here $(L)_{>0}$ (resp. $\left.(L)_{<0}\right)$ is the strictly upper (resp. lower) triangular matrix of $L$.

Any equation in the Lax form $\frac{d}{d t} L=[B, L]$ for matrices $L$ and $B$ has the immediate consequence that the flow preserves the spectrum of $L$. To check this, it suffices to show that the function $\operatorname{tr}\left(L^{k}\right)$, the trace of $L^{k}$, is constant for each $k$. One shows first by induction that $\frac{d}{d t} L^{k}=\left[B, L^{k}\right]$ and then observes that $\frac{d}{d t}\left[\operatorname{tr}\left(L^{k}\right)\right]=\operatorname{tr}\left[\frac{d}{d t}\left(L^{k}\right)\right]=\operatorname{tr}\left[B, L^{k}\right]=0$. We now have $n-1$ independent invariant functions

$$
H_{k}(L)=\frac{1}{k+1} \operatorname{tr} L^{k+1} .
$$

The Hamiltonian (2.1) is related to $H_{1}(L)$ by $H=4 H_{1}(L)$ with the change of variables (2.4).

A property of real tridiagonal symmetric matrices (2.7) with $a_{k} \neq 0$ for all $k$ is that the eigenvalues $\lambda_{k}$ are real and distinct. Let $\Lambda$ be a set of $n$ real distinct eigenvalues, and let $\mathcal{M}_{\Lambda}=\{L$ in (2.7) : $\operatorname{spec}(L)=\Lambda\}$. Then $\mathcal{M}_{\Lambda}$ is a symplectic manifold. Each invariant function $H_{k}(L)$ generates a Hamiltonian flow via the symplectic structure, and the flows are involutive with respect to that structure (see [5] for the general framework and [26] for the Toda lattice specifically). In Section 2.5. we describe the Lie-Poisson structure for the Equations (2.6).

Moser [59] analyzes the dynamics of the Toda particles, showing that for any initial configuration, $q_{k+1}-q_{k}$ tends to $\infty$ as $t \rightarrow \pm \infty$. Thus, the off-diagonal entries of $L$ tend to zero as $t \rightarrow \pm \infty$ so that $L$ tends to a diagonal matrix whose diagonal entries are the eigenvalues. We will order them as $\lambda_{1}<\lambda_{2}<\cdots<\lambda_{n}$. The analysis in [59] shows the sorting property of the eigenvalues,

$$
L(t) \longrightarrow \begin{cases}\operatorname{diag}\left(\lambda_{n}, \lambda_{n-1}, \cdots, \lambda_{1}\right) & \text { as } t \rightarrow \infty, \\ \operatorname{diag}\left(\lambda_{1}, \lambda_{2}, \cdots, \lambda_{n}\right) & \text { as } t \rightarrow-\infty .\end{cases}
$$

The physical interpretation of this is that as $t \rightarrow-\infty$, the particles $q_{k}$ approach the velocities $p_{k}(-\infty)=-2 \lambda_{k}$, and as $t \rightarrow \infty$, the velocities are interchanged so that $p_{k}(\infty)=-2 \lambda_{n-k+1}$. Asymptotically, the trajectories behave as

$$
\left\{\begin{array}{l}
q_{k}(t) \approx \lambda_{k}^{ \pm} t+c_{k}^{ \pm}, \\
p_{k}(t) \approx \lambda_{k}^{ \pm},
\end{array} \quad \text { as } t \rightarrow \pm \infty .\right.
$$


where $\lambda_{k}^{+}=\lambda_{k}$ and $\lambda_{k}^{-}=\lambda_{n-k+1}$.

Symes solves the Toda lattice using the QR-factorization; his solution, which he verifies in 72 and proves in a more general context in [71, is equivalent to the following. To solve (2.5) with initial matrix $L(0)$, take the exponential $e^{t L(0)}$ and use Gram-Schmidt orthonormalization to factor it as

$$
e^{t L(0)}=k(t) r(t),
$$

where $k(t) \in S O(n)$ and $r(t)$ is upper triangular. Then the solution of (2.5) is

$$
L(t)=k^{-1}(t) L(0) k(t)=r(t) L(0) r^{-1}(t) .
$$

Since the Gram-Schmidt orthonormalization of $e^{t L(0)}$ can be done for all $t$, this shows that the solution of the Toda lattice equations (2.5) on the set of symmetric tridiagonal matrices $L$ of (2.7) is defined for all $t$.

We mention also the $\tau$-functions, which play a key role of the theory of integrable systems (see for example [36, 58]). Let us first introduce the following symmetric matrix, called the moment matrix,

$$
M(t):=e^{2 t L(0)}=r^{T}(t) k^{T}(t) k(t) r(t)=r^{T}(t) r(t),
$$

where $r^{T}$ denotes the transpose of $r$, and note that $k^{T}=k^{-1}$. The decomposition of a symmetric matrix to an upper-triangular matrix times its transpose on the left is called the Cholesky factorization. This factorization is used to find the matrix $r$, and then the matrix $k$ can be found by $k=e^{t L(0)} r^{-1}$. The $\tau$-functions, $\tau_{j}$ for $j=1, \ldots, n-1$, are defined by

$$
\tau_{j}(t):=\operatorname{det}\left(M_{j}(t)\right)=\prod_{i=1}^{j} r_{i}(t)^{2},
$$

where $M_{j}$ is the $j \times j$ upper-left submatrix of $M$, and we denote $\operatorname{diag}(r)=\operatorname{diag}\left(r_{1} \ldots, r_{n}\right)$. We see from (2.10), i.e. $L(t) r(t)=r(t) L(0)$, that we have

$$
a_{j}(t)=a_{j}(0) \frac{r_{k+1}(t)}{r_{k}(t)} .
$$

Since $r_{k}(t) \neq 0$ for all $k$, the signs $a_{j}(t)$ remain the same. With (2.12) and (2.5), we obtain

$$
a_{j}(t)=a_{j}(0) \frac{\sqrt{\tau_{j+1}(t) \tau_{j-1}(t)}}{\tau_{j}(t)} \quad \text { and } \quad b_{j}(t)=\frac{1}{2} \frac{d}{d t} \ln \left(\frac{\tau_{j}(t)}{\tau_{j-1}(t)}\right) .
$$

One should note that the $\tau$-functions are just defined from the moment matrix $M=e^{2 t L(0)}$, and the solutions $\left(a_{j}(t), b_{j}(t)\right)$ are explicitly given by those $\tau$-functions without the factorization.

2.2. Hessenberg form. The symmetric matrix $L$ in (2.7), when conjugated by the diagonal matrix $D=\operatorname{diag}\left(1, a_{1}, \ldots, a_{n-1}\right)$, gives a matrix $Y=D L D^{-1}$ in Hessenberg form:

$$
Y=\left(\begin{array}{cccc}
b_{1} & 1 & & \\
a_{1}^{2} & \ddots & \ddots & \\
& \ddots & \ddots & 1 \\
& & a_{n-1}^{2} & b_{n}
\end{array}\right) .
$$

The Toda equations (2.6) now take the Lax form for $X:=2 Y$,

$$
\frac{d}{d t} X=\left[X,(X)_{<0}\right]=\left[(X)_{\geq 0}, X\right]
$$


where $(X)_{\geq 0}=X-(X)_{<0}$ is the upper-triangular part of $X$. Equation (2.15) with

$$
X=\left(\begin{array}{cccc}
f_{1} & 1 & & \\
g_{1} & \ddots & \ddots & \\
& \ddots & \ddots & 1 \\
& & g_{n-1} & f_{n}
\end{array}\right)
$$

is called the Hessenberg form of the nonperiodic Toda lattice. Again, since the equations are in Lax form, the functions $H_{k}(X)=\frac{1}{k+1} \operatorname{tr} X^{k+1}$ are constant in $t$.

Notice that the Hessenberg and symmetric Lax formulations of (2.3) are simply different ways of expressing the same system. The solutions exist for all time and exhibit the same behavior as $t \rightarrow \pm \infty$. However, when we allow the subdiagonal entries to assume any real value, the symmetric and Hessenberg forms differ in their geometry and topology and in the character of their solutions.

2.3. Isospectral manifolds in the real tridiagonal symmetric form. Here we consider the Lax equation (2.6) where the $a_{k}$ in the symmetric Lax matrix $L$ may be any real numbers. As mentioned in Remark 2.1, the equations with different signs in the $a_{k}$ are the same. In particular, if $a_{k} \neq 0$ for all $k$, then the eigenvalues are real and distinct.

Let $\mathcal{M}_{\Lambda}$ denote the set of $n \times n$ matrices of the form (2.7) with fixed eigenvalues $\lambda_{1}<\lambda_{2}<$ $\cdots<\lambda_{n} . \mathcal{M}_{\Lambda}$ contains $2^{n-1}$ components of dimension $n-1$, where each component consists of all matrices in $\mathcal{M}_{\Lambda}$ with a fixed choice of sign for each $a_{k}$. The solution of (2.6) with initial condition in a given component remains in that component for all $t$, because the solutions preserve the sign of each $a_{k}$. Each lower-dimensional component, where one or more $a_{k}$ is zero and the signs of the other $a_{k}$ are fixed, is also preserved by the Toda flow through any initial matrix in that component. Tomei [75] shows that $\mathcal{M}_{\Lambda}$ is a compact smooth manifold of dimension $n-1$ containing $2^{n-1}$ open components, each diffeomorphic to $\mathbb{R}^{n-1}$ (see also [7]). On each component, $a_{k} \neq 0$ for all $k$, and the sign of each $a_{k}$ is fixed. The components are glued together along the lower-dimensional sets where one or more $a_{k}$ is zero.

For $n=3$, there are four 2-dimensional components, denoted as $\mathcal{M}_{++}, \mathcal{M}_{+-}, M_{-+}$, and $\mathcal{M}_{--}$, according to the signs of $a_{1}$ and $a_{2}$. The closure of each component is obtained by adjoining the six diagonal matrices $\operatorname{diag}\left(\lambda_{i}, \lambda_{j}, \lambda_{k}\right)$ where all the $a_{k}$ vanish (these are the fixed points of the Toda flows) and six 1-dimensional sets where exactly one $a_{k}$ is zero. The four principal components are glued together along the loci of $\mathcal{M}_{\Lambda}$ where one or more $a_{k}$ vanish. In Figure 2.1, we illustrate the Tomei manifold $\mathcal{M}_{\Lambda}$ for the $\mathfrak{s l}_{3}(\mathbb{R})$ symmetric Toda lattice,

$$
\mathcal{M}_{\Lambda}=\overline{\mathcal{M}}_{++} \cup \overline{\mathcal{M}}_{+-} \cup \overline{\mathcal{M}}_{-+} \cup \overline{\mathcal{M}}_{--},
$$

where the cups include the specific gluing according to the signs of the $a_{k}$. The resulting manifold $\mathcal{M}_{\Lambda}$ is a connected sum of two tori, the compact Riemann surface of genus two. This can be easily seen from Figure 2.1 as follows: Gluing those four hexagons, $\mathcal{M}_{\Lambda}$ consists of 6 vertices, 12 edges and 4 faces. Hence the Euler characteristic is given by $\chi\left(\mathcal{M}_{\Lambda}\right)=6-12+4=-2$, which implies that the manifold has genus $g=2$ (recall $\chi=2-2 g$ ). The fact that $\mathcal{M}_{\Lambda}$ is orientable can be shown by giving an orientation for each hexagon so that the directions of two edges in the gluing cancel each other. Since compact two-dimensional surfaces are completely characterized by their orientability and Euler characters, we conclude that the manifold $\mathcal{M}_{\Lambda}$ is a connected sum of two tori.

The Euler characteristic of $\mathcal{M}_{\Lambda}$ (for general $n$ ) is determined in [75] as follows. Let $L=$ $\operatorname{diag}\left(\lambda_{\sigma(1)}, \ldots, \lambda_{\sigma(n)}\right)$ be a diagonal matrix in $\mathcal{M}_{\Lambda}$, where $\sigma$ is a permutation of the numbers $\{1, \ldots, n\}$, and let $r(L)$ be the number of times that $\sigma(k)$ is less than $\sigma(k+1)$. Denote by $E(n, k)$ the number of diagonal matrices in $\mathcal{M}_{\Lambda}$ with $r(L)=k$. Then the Euler characteristic of $\mathcal{M}_{\Lambda}$ is the alternating 


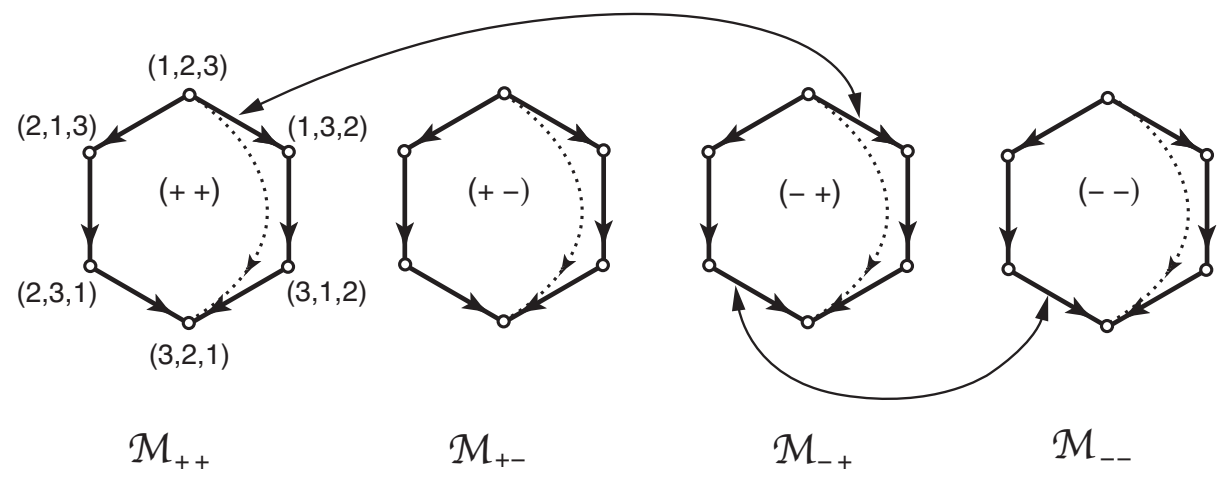

Figure 2.1. The Tomei manifold $\mathcal{M}_{\Lambda}$ for the symmetric tridiagonal $\mathfrak{s l}_{3}(\mathbb{R})$ Toda lattice. The 3 -tuples $(i, j, k)$ on the vertices indicate the diagonal matrices $L=$ $\operatorname{diag}\left(\lambda_{i}, \lambda_{j}, \lambda_{k}\right)$. Each hexagon $\mathcal{M}_{\epsilon_{1}, \epsilon_{2}}$ corresponds to the moment polytope (see Section 3.1) for the Toda lattice with the signs $\left(\epsilon_{1}, \epsilon_{2}\right)=\left(\operatorname{sgn}\left(a_{1}\right), \operatorname{sgn}\left(a_{2}\right)\right)$. The boundaries correspond to the $\operatorname{sl}(2, \mathbb{R})$ Toda lattices associated with either $a_{1}=0$ or $a_{2}=0 . \mathcal{M}_{\Lambda}$ results from gluing corresponding edges of the hexagons. For example, the edge between $(1,2,3)$ and $(1,3,2)$ in $\mathcal{M}_{++}$is glued with the same edge in $\mathcal{M}_{-+}$, since this edge indicates $a_{1}=0$ and $a_{2}>0$. In the other gluing shown, $a_{2}=0$ and $a_{1}<0$.

sum of the $E(n, k)$ :

$$
\chi\left(\mathcal{M}_{\Lambda}\right)=\sum_{k=0}^{n}(-1)^{k} E(n, k) .
$$

An isospectral set where the eigenvalues are not distinct is not a manifold. For example, the isospectral set with spectrum $(1,1,3)$ has the shape of a figure eight [75, 13].

2.4. Indefinite Toda lattice in real tridiagonal Hessenberg form. We return to the Hessenberg form with $X$ as in (2.16), and allow the $g_{k}$ to assume arbitrary real values. Recall that in the formulation of the original Toda equations, all the $g_{k}$ were positive, so that the eigenvalues were real and distinct. When $g_{k} \neq 0$ for some $k$, the eigenvalues may be complex or may coincide. Even in the case where all the eigenvalues are real and distinct, the case with some $g_{k}<0$ causes blow-ups in the flows so that the topology of the isospectral manifolds is very different from the topology of the Tomei manifolds described in the previous section [46, 23].

The matrices of the form (2.16) with $g_{k} \neq 0$ for all $k$ are partitioned into $2^{n-1}$ different Hamiltonian systems, each determined by a choice of signs of the $g_{k}$. Letting $\sigma_{k}= \pm 1$ for $k=1, \ldots, n$ and taking the sign of $g_{k}$ to be $\sigma_{k} \sigma_{k+1}$, Kodama and Ye [45] give the Hamiltonian for the system with this choice of signs as

$$
H=\frac{1}{2} \sum_{k=1}^{n} y_{k}^{2}+\sum_{k=1}^{n-1} \sigma_{k} \sigma_{k+1} e^{-\left(x_{k+1}-x_{k}\right)},
$$

where the variables $\left(f_{k}, g_{k}\right)$ in the Hessenberg form are given by

$$
\left\{\begin{aligned}
f_{k} & =-\frac{1}{2} y_{k}, & k & =1, \ldots, n \\
g_{k} & =\frac{1}{4} \sigma_{k} \sigma_{k+1} e^{-\left(x_{k+1}-x_{k}\right)}, & k & =1, \ldots, n-1 .
\end{aligned}\right.
$$


The system (2.15) with the Hamiltonian $H$ in (2.17) is called the indefinite Toda lattice. The negative signs in (2.17) correspond to attractive forces between adjacent particles, which causes the system to become undefined at finite values of $t$, as is seen in the solutions obtained in [45] and [46] by inverse scattering.

The blow-ups in the solutions are also apparent in the factorization solution of the Hessenberg form. To solve (2.15) with initial condition $X(0)$, we consider the LU-factorization of the exponential $e^{t X(0)}$,

$$
e^{t X(0)}=n(t) b(t)
$$

where $n(t)$ is lower unipotent and $b(t)$ is upper-triangular. Then, as shown in [62, 61] (see also [34, 60]),

$$
X(t)=n^{-1}(t) X(0) n(t)=b(t) X(0) b^{-1}(t)
$$

solves (2.15). Notice that the factorization (2.19) is obtained by Gaussian elimination, which multiplies $e^{t X(0)}$ on the left by elementary row operations to put it in upper-triangular form. This process works only when all principal minors (the determinants of upper left $k \times k$ blocks, which are the $\tau$-functions as defined in (2.12) ) are nonzero. At particular values of $t \in \mathbb{R}$, this factorization can fail, and the solution (2.20) becomes undefined.

The solutions $\left(f_{k}, g_{k}\right)$ can be expressed in terms of the $\tau$-functions

$$
\tau_{k}(t):=\left[e^{t X(0)}\right]_{k}=\prod_{j=1}^{k} d_{j}(t),
$$

where $\left[e^{t X(0)}\right]_{k}$ is the $k \times k$ principal minor of $e^{t X(0)}$, and $\operatorname{diag}(b)=\operatorname{diag}\left(d_{1}, \ldots, d_{n}\right)$. As in the previous case of symmetric Toda, from (2.20), we have

$$
g_{k}(t)=g_{k}(0) \frac{\tau_{k+1}(t) \tau_{k-1}(t)}{\tau_{k}(t)^{2}} \quad \text { and } \quad f_{k}(t)=\frac{d}{d t} \ln \left(\frac{\tau_{k}(t)}{\tau_{k-1}(t)}\right) .
$$

Now it it clear that the factorization (2.19) fails if and only if $\tau_{k}(t)=0$ for some $k$. Then a blow-up (singularity) of the system (2.15) can be characterized by the zero sets of the $\tau$-functions.

Example 2.2. To see how blow-ups occur in the factorization solution, consider the initial matrix

$$
X_{0}=\left(\begin{array}{rr}
1 & 1 \\
-1 & -1
\end{array}\right)
$$

When $t \neq-1$,

$$
e^{t X_{0}}=\left(\begin{array}{cc}
1+t & t \\
-t & 1-t
\end{array}\right)=\left(\begin{array}{cc}
1 & 0 \\
\frac{-t}{1+t} & 1
\end{array}\right)\left(\begin{array}{cc}
1+t & t \\
0 & \frac{1}{1+t}
\end{array}\right)
$$

and the solution evolves as in (2.20). The $\tau$-function is given by $\tau_{1}(t)=1+t$, and when $t=-1$, this factorization does not work. However, we can multiply $e^{-X_{0}}$ on the left by a lower unipotent matrix $n^{-1}$ (in this case the identity) to put it in the form $w b$, where $w$ is a permutation matrix:

$$
e^{-X_{0}}=\left(\begin{array}{cc}
0 & -1 \\
1 & 2
\end{array}\right)=\left(\begin{array}{ll}
1 & 0 \\
0 & 1
\end{array}\right)\left(\begin{array}{cc}
0 & -1 \\
1 & 0
\end{array}\right)\left(\begin{array}{ll}
1 & 2 \\
0 & 1
\end{array}\right) .
$$

This example will be taken up again in Section 3.2, where it is shown how the factorization using a permutation matrix leads to a compactification of the flows.

In general, when the factorization (2.19) is not possible at time $t=\bar{t}, e^{\overline{t X}(0)}$ can be factored as $e^{\bar{t} X(0)}=n(\bar{t}) w b(\bar{t})$ for some permutation matrix $w$. In [21, this factorization is used to complete the flows (2.20) through the blow-up times by embedding them into a flag variety. We examine this further in the context of the complex tridiagonal Hessenberg form. 
To describe the topology of a generic isospectral set $\mathcal{M}_{\Lambda}$ in this version of the Toda lattice, it is first shown that because of the blow-ups in $X, \mathcal{M}_{\Lambda}$ is a noncompact manifold of dimension $n-1$ [46. The manifold is compactified by completing the flows through the blow-up times. The $2 \times 2$ case is basic to the compactification for general $n$. The set of $2 \times 2$ matrices with fixed eigenvalues $\lambda_{1}<\lambda_{2}$,

$$
\mathcal{M}_{\Lambda}=\left\{\left(\begin{array}{cc}
f_{1} & 1 \\
g_{1} & f_{2}
\end{array}\right): \lambda_{1}<\lambda_{2}\right\},
$$

consists of two components, $\mathcal{M}_{+}$with $g_{1}>0$ and $\mathcal{M}_{-}$with $g_{1}<0$, together with two fixed points,

$$
X_{1}=\left(\begin{array}{cc}
\lambda_{1} & 1 \\
0 & \lambda_{2}
\end{array}\right) \text { and } X_{2}=\left(\begin{array}{cc}
\lambda_{2} & 1 \\
0 & \lambda_{1}
\end{array}\right) \text {. }
$$

Writing $f_{2}=\lambda_{1}+\lambda_{2}-f_{1}$ and substituting this into the equation for the determinant, $f_{1} f_{2}-g_{1}=\lambda_{1} \lambda_{2}$, shows that $M_{\lambda}$ is the parabola

$$
g_{1}=-\left(f_{1}-\lambda_{1}\right)\left(f_{1}-\lambda_{2}\right) .
$$

This parabola opens down, crossing the axis $g_{1}=0$ at $f_{1}=\lambda_{1}$ and $f_{1}=\lambda_{2}$, corresponding to the fixed points $X_{1}$ and $X_{2}$. For an initial condition with $g_{1}>0$, the solution is defined for all $t$; it flows away from $p_{2}$ toward $p_{1}$. This illustrates what is known as the sorting property, which says that as $t \rightarrow \infty$, the flow tends toward the fixed point with the eigenvalues in decreasing order along the diagonal. The component with $g_{1}<0$ is separated into disjoint parts, one with $f_{1}<\lambda_{1}$ and the other with $f_{1}>\lambda_{2}$. The solution starting at an initial matrix with $f_{1}>\lambda_{2}$ flows toward the fixed point $X_{2}$ as $t \rightarrow \infty$. For an initial matrix with $f_{1}<\lambda_{1}$, the solution flows away from $X_{1}$, blowing up at a finite value of $t$. By adjoining a point at infinity to connect these two branches of the parabola, the flow is completed through the blow-up time and the resulting manifold is the circle, $S^{1}$.

For general $n$, the manifold $\mathcal{M}_{\Lambda}$ with spectrum $\Lambda$ contains $n$ ! fixed points of the flow, where the eigenvalues are arranged along the diagonal. These vertices are connected to each other by incoming and outgoing edges analogous to the flows connecting the two vertices when $n=2$. The result is nonorientable for $n>2$. For $n=3$, it is a connected sum of two Klein bottles. Figure 2.2 illustrates the compactification of $\mathcal{M}_{\Lambda}$ for the $\mathfrak{s l}_{3}(\mathbb{R})$ indefinite Toda lattice. With this gluing, the compactified manifold $\overline{\mathcal{M}}_{\Lambda}$ has Euler characteristic $\chi\left(\overline{\mathcal{M}}_{\Lambda}\right)=-2$ as in the case of the Tomei manifold (see Figure 2.1). The non-orientability is seen in the non-cancellation of the given orientations of the hexagons.

Casian and Kodama [10] (see also [12]) show that the compactified isospectral manifold is identified as a connected completion of the disconnected Cartan subgroup of $G=\operatorname{Ad}\left(S L(n, \mathbb{R})^{ \pm}\right)$. It is diffeomorphic to a toric variety in the flag variety of $G$. We give more details in Section 6.2.

2.5. Full symmetric real Toda lattice. In 1986, the paper [18 by Deift, Li, Nanda, and Tomei brought a radical departure from the tridiagonal Toda lattices that had been heretofore studied, by expanding the phase space to the set of full symmetric matrices. Consider the symmetric Toda equation

$$
\frac{d}{d t} L=\left[\Pi_{\mathfrak{s o}}(L), L\right] \quad \text { with } \quad \Pi_{\mathfrak{s o}}(L)=(L)_{>0}-(L)_{<0} .
$$

as in (2.6), where $L$ is now a full symmetric matrix with distinct eigenvalues. The authors of 18 show that (2.25) remains completely integrable for the generic case. They present a sufficient number of constants of motion in involution and construct the associated angle variables. The additional constants of motion are found by a chopping construction on the matrix that was later extended in 22. to the complex full Kostant-Toda lattice, which we describe in more detail in Section 3.3 . 


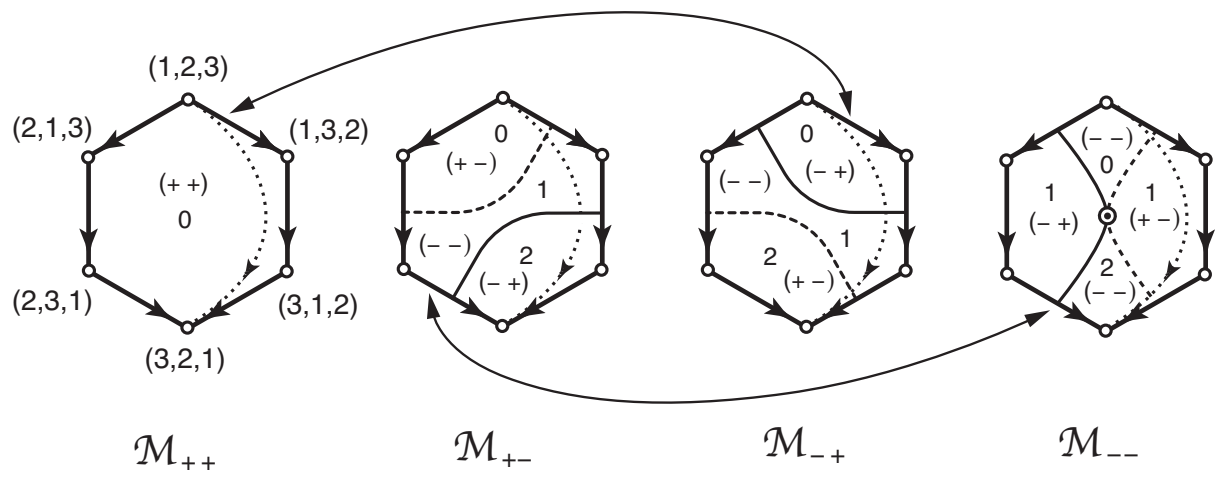

Figure 2.2. The compactification of the isospectral manifold $\mathcal{M}_{\Lambda}$ for the indefinite $\mathfrak{s l}_{3}(\mathbb{R})$ Toda lattice. Each hexagon indicates the moment polytope associated with an indefinite Toda lattice. The signs $\left(\epsilon_{1}, \epsilon_{2}\right)$ in $\mathcal{M}_{\epsilon_{1}, \epsilon_{2}}$ are those of $\left(g_{1}, g_{2}\right)$ as $t \rightarrow-\infty$, and the signs in the hexagons indicate the signs of $\left(g_{1}, g_{2}\right)$. The gluing rule according to the sign changes of $g_{i}$ is the same as that in the Tomei manifold, but the pattern is now different. For example, the edge between $(2,3,1)$ and $(3,2,1)$ in $\mathcal{M}_{--}$is now glued with that in $\mathcal{M}_{+-}$. The solid and dashed lines in the hexagons show the points where the solutions blow up; $\tau_{1}=0$ (solid) and $\tau_{2}=0$ (dashed). The numbers in the sections indicate the number of blow-ups along the flow from $t=-\infty$ to $+\infty$ (see Section 6.2).

The Lie-Poisson structure on the space of symmetric real matrices is the Kostant-Kirillov form (as explained in [18]), with respect to which the Toda flows (2.25) may be expressed in Hamiltonian form as

$$
\frac{d}{d t} L=\left\{H_{1}, L\right\}(L) \quad \text { with } \quad H_{1}(L)=\frac{1}{2} \operatorname{tr}\left(L^{2}\right) .
$$

Using the Poisson structure, we may extend (2.25) to define the Toda lattice hierarchy generated by the Hamiltonians $H_{k}(L)=\frac{1}{k+1} \operatorname{tr}\left(L^{k+1}\right)$ :

$$
\frac{\partial}{\partial t_{k}} L=\left\{H_{k}, L\right\}(L)=\left[\Pi_{\mathfrak{s o}} \nabla H_{k}, L\right] \quad \text { for } \quad k=1,2, \ldots, n-1 .
$$

where $\operatorname{tr}(X \nabla f)=\lim _{\epsilon \rightarrow 0} \frac{d}{d \epsilon} f(L+\epsilon X)$ so that $\nabla H_{k}=L^{k}$. Each flow stays on a co-adjoint orbit in the phase space of the symmetric Toda, which is $\operatorname{Sym}(n):=\left\{L \in \mathfrak{s l}_{n}(\mathbb{R}): L^{T}=L\right\}$. The Poisson structure is nondegenerate when restricted to a co-adjoint orbit, and the level sets of the integrals found in [18] are the generic co-adjoint orbits.

In [42, Kodama and McLaughlin give the explicit solution of the Toda lattice hierarchy (2.26) on full symmetric matrices with distinct eigenvalues by solving the inverse scattering problem of the system

$$
L \Phi=\Phi \Lambda \quad \text { and } \quad \frac{\partial}{\partial t_{k}} \Phi=\Pi_{\mathfrak{s o}}\left(L^{k}\right) \Phi
$$

with $\Lambda=\operatorname{diag}\left(\lambda_{1}, \ldots, \lambda_{n}\right)$. Since $L$ is symmetric, the matrix $\Phi$ of eigenvectors is taken to be orthogonal:

$$
L=\Phi \Lambda \Phi^{T}
$$

with $\Phi=\left[\phi\left(\lambda_{1}\right), \ldots, \phi\left(\lambda_{n}\right)\right]$, where the $\phi\left(\lambda_{k}\right)$ is the normalized eigenvector of $L$ with eigenvalue $\lambda_{k}$.

The indefinite extension of the full symmetric Toda lattice is studied in 42, where explicit solutions of $\phi\left(\lambda_{k}, t\right)$ are obtained by inverse scattering. The authors also give an alternative derivation 
of the solution using the factorization method of Symes [72, where $e^{t L(0)}$ is factored into a product of a pseudo-orthogonal matrix times an upper triangular matrix (the HR-factorization).

\section{Complex Toda lattices}

The jump from real to complex Toda lattices brought with it a powerful new tool for understanding the geometry of the iso-spectral varieties, namely, embeddings of the isospectral sets into the flag varieties. Under these mappings, the Toda flows generate group actions and blow-ups are compactified. The geometry and topology of the compactified isospectral sets can then be described in terms of the moment map and moment polytope of the flag variety.

3.1. The moment map. Let $G$ be a complex semisimple Lie group, $H$ a Cartan subgroup of $G$, and $B$ a Borel subgroup containing $H$. If $P$ is a parabolic subgroup of $G$ that contains $B$, then $G / P$ can be realized as the orbit of $G$ through the projectivized highest weight vector in the projectivization, $\mathbb{P}(V)$, of an irreducible representation $V$ of $G$. Let $\mathcal{A}$ be the set of weights of $V$, counted with multiplicity; the weights belong to $\mathfrak{h}_{\mathbb{R}}^{*}$, the real part of the dual of the Lie algebra $\mathfrak{h}$ of $H$. Let $\left\{v_{\alpha}: \alpha \in \mathcal{A}\right\}$ be a basis of $V$ consisting of weight vectors. A point $[\mathrm{X}]$ in $G / P$, represented by $X \in V$, has homogeneous coordinates $\pi_{\alpha}(X)$, where $X=\sum_{\alpha \in \mathcal{A}} \pi_{\alpha}(X) v_{\alpha}$. The moment map as defined in 39] sends $G / P$ into $\mathfrak{h}_{\mathbb{R}}^{*}$ :

$$
\begin{aligned}
& \mu: G / P \quad \longrightarrow \quad \mathfrak{h}_{\mathbb{R}}^{*} \\
& {[X] \longmapsto \frac{\sum_{\alpha \in \mathcal{A}}\left|\pi_{\alpha}(X)\right|^{2} \alpha}{\sum_{\alpha \in \mathcal{A}}\left|\pi_{\alpha}(X)\right|^{2}}}
\end{aligned}
$$

Its image is the weight polytope of $V$, also referred to as the moment polytope of $G / P$.

The fixed points of $H$ in $G / P$ are the points in the orbit of the Weyl group $W$ through the projectivized highest weight vector of $V$; they correspond to the vertices of the polytope under the moment map. Let $\overline{H \cdot[X]}$ be the closure of the orbit of $H$ through $[X]$. Its image under $\mu$ is the convex hull of the vertices corresponding to the fixed points contained in $\overline{H \cdot[X]}$; these vertices are the weights $\left\{\alpha \in W \cdot \alpha^{V}: \pi_{\alpha}(X) \neq 0\right\}$, where $\alpha^{V}$ is the highest weight of $V[\underline{6}$. In particular, the image of a generic orbit, where no $\pi_{\alpha}$ vanishes, is the full polytope. The real dimension of the image is equal to the complex dimension of the orbit.

For $G=S L(n, \mathbb{C}), B$ the upper triangular subgroup, and $H$ the diagonal torus. The choice of $B$ determines a splitting of the root system into positive and negative roots and a system $\Delta$ of simple roots. The simple roots are $\mathrm{L}_{i}-\mathrm{L}_{i+1}$, where $i=1, \ldots, n-1$ and $\mathrm{L}_{i}$ is a weight of the standard representation of $\mathfrak{s l}_{n}$, i.e. for $h=\operatorname{diag}\left(h_{1}, \ldots, h_{n}\right) \in \mathfrak{h}, \mathrm{L}(h)=h_{i}$. Then let $\mathfrak{h}_{\mathbb{R}}^{*}$ denote the dual of $\mathfrak{h}$,

$$
\mathfrak{h}_{\mathbb{R}}^{*}:=\operatorname{Span}_{\mathbb{R}}\left\{\mathrm{L}_{1}, \ldots, \mathrm{L}_{n} \mid \sum_{j=1}^{n} \mathrm{~L}_{j}=0\right\} \cong \mathbb{R}^{n-1} .
$$

The Weyl group $W=\mathcal{S}_{n}$ acts by permuting the weight $\mathrm{L}_{i}$, and the moment polytope of $G / B$ is the convex hull of the weights $\mathrm{L}_{i_{1}, \ldots, i_{n}}$ for $\left(i_{1}, \ldots, i_{n}\right)=\pi(1, \ldots, n)$ with $\pi \in \mathcal{S}_{n}$, which is given by

$$
\mathrm{L}_{i_{1}, \ldots, i_{n}}:=(n-1) \mathrm{L}_{i_{1}}+(n-2) \mathrm{L}_{i_{2}}+\cdots+\mathrm{L}_{i_{n-1}} .
$$

where the highest weight is $L_{1,2, \ldots, n}$. This moment polytope is referred to as the permutohedron, which is given by

$$
\operatorname{Perm}_{n}=\operatorname{Conv}\left\{\mathrm{L}_{\pi(1, \ldots, n)} \in \mathfrak{h}_{\mathbb{R}}^{*} \mid \pi \in \mathcal{S}_{n}\right\}
$$


3.2. Complex tridiagonal Hessenberg form. Let $\mathcal{M}$ be the set of complex tridiagonal Hessenberg matrices of the form (2.16), where the $f_{k}$ and $g_{k}$ are arbitrary complex numbers. As before, the Toda flow is defined by (2.15) and the eigenvalues (equivalently, the traces of the powers of $X$ ) are constants of motion. The Hamiltonian $H_{k}(X)=\frac{1}{k+1} \operatorname{tr}\left(X^{k+1}\right)$ generates the flow

$$
\frac{\partial X}{\partial t_{k}}=\left[X,\left(\nabla H_{k}\right)_{<0}\right]=\left[X,\left(X^{k}\right)_{<0}\right] .
$$

The solution of (3.5) can be found by the LU-factorization as in (2.19). That is, with $e^{t_{k} X^{k}(0)}=$ $n\left(t_{k}\right) b\left(t_{k}\right)$, we have Then

$$
X\left(t_{k}\right)=n^{-1}\left(t_{k}\right) X(0) n\left(t_{k}\right)=b\left(t_{k}\right) X(0) b^{-1}\left(t_{k}\right) .
$$

Fix the eigenvalues $\lambda_{j}$, and consider the level set $\mathcal{M}_{\Lambda}$ consisting of all matrices in $\mathcal{M}$ with spectrum $\Lambda=\left\{\lambda_{1}, \ldots, \lambda_{n}\right\}$ (we often identify $\Lambda=\operatorname{diag}\left(\lambda_{1}, \ldots, \lambda\right)$. In contrast to the real tridiagonal flows described in Section 2.4 when $X$ is complex, $\mathcal{M}_{\Lambda}$ is no longer partitioned by signs of the $g_{k}$. There is only one maximal component where no $g_{k}$ vanishes. The $n-1$ flows through any initial $X$ with $g_{k} \neq 0$ for all $k$ generates the whole component.

In the case of distinct eigenvalues, Ercolani, Flaschka and Haine in 21] construct a minimal nonsingular compactification of $\mathcal{M}_{\Lambda}$ on which the flows (3.5) extend to global holomorphic flows. The compactification is induced by an embedding of $\mathcal{M}_{\Lambda}$ into the flag variety $S L(n, \mathbb{C}) / B$ with $B$, the set of weakly upper-triangular matrices. The embedding depends on the following factorization by Kostant [52] of $X \in \mathcal{M}_{\Lambda}$. Let $\epsilon_{\Lambda}$ be the matrix with $\left(\lambda_{1}, \ldots, \lambda_{n}\right)$ on the diagonal, 1's on the superdiagonal, and 0's elsewhere. Then every $X \in \mathcal{M}_{\Lambda}$ can be conjugated to $\epsilon_{\Lambda}$ by a unique element $n \in N$, the set of lower-triangular unipotent matrices:

$$
X=n \epsilon_{\Lambda} n^{-1} .
$$

This defines a map of $\mathcal{M}_{\Lambda}$ into $G / B$ :

$$
\begin{aligned}
& j_{\Lambda}: \mathcal{M}_{\Lambda} \rightarrow G / B \\
& X \mapsto n^{-1} \bmod B \text {. }
\end{aligned}
$$

This mapping is an embedding [51, and the closure, $\overline{j_{\Lambda}\left(\mathcal{M}_{\Lambda}\right)}$, of its image is a nonsingular and minimal compactification of $\mathcal{M}_{\Lambda}$. Let $n_{0}$ be the unique lower unipotent matrix such that $X(0)=$ $n_{0} \epsilon_{\Lambda} n_{0}^{-1}$. Then the solution (3.6) is $X\left(t_{k}\right)=n^{-1}\left(t_{k}\right) n_{0} \epsilon_{\Lambda} n_{0}^{-1} n\left(t_{k}\right)$, where $n_{0}^{-1} n\left(t_{k}\right)$ is lower unipotent. The Toda flow $X\left(t_{k}\right)$ is mapped into the flag variety as

$$
\begin{aligned}
j_{\Lambda}\left(X\left(t_{k}\right)\right) & =u_{0}^{-1} n\left(t_{k}\right) \bmod B \\
& =u_{0}^{-1} e^{t_{k} X^{k}(0)} \bmod B .
\end{aligned}
$$

Even at values of $t_{k}$ where the first expression in (3.9) is not defined because the LU-factorization of $e^{t_{k} X^{k}(0)}$ is not possible, the second expression in (3.9) is defined. In this way, the embedding of $X\left(t_{k}\right)$ into $G / B$ completes the flows through the blow-up times. This used in [21] to study the nature of the blow-ups of $X\left(t_{k}\right)$.

To illustrate this in a simple case, consider Example 2.2 from Section 2.4 The isospectral set of $2 \times 2$ Hessenberg matrices with both eigenvalues zero is embedded into the flag variety $S L(2, \mathbb{C}) / B$, which has the cell decomposition

$$
S L(2, \mathbb{C}) / B=N B / B \sqcup N\left(\begin{array}{cc}
0 & -1 \\
1 & 0
\end{array}\right) B / B .
$$

The big cell, $N B / B$, contains the image of the flow $X(t)$ whenever this flow is defined, that is, whenever the factorization $e^{t X_{0}}=n(t) b(t)$ is possible. At $t=-1$, where $X(t)$ is undefined, the 
embedding $j_{\Lambda}$ completes the flow through the singularity. The image $j_{\Lambda}(X(t))$ passes through the flag $u_{0}^{-1} e^{-X(0)}$ at time $t=-1$, which is the cell on the right in (3.10).

The cell decomposition (3.10) is a special case of the cell stratification of $G / B$ known as the Bruhat decomposition. This decomposition is defined in terms of the Weyl group $W$ as

$$
G / B=\bigsqcup_{w \in W} N \dot{w} B / B
$$

where each component $N \dot{w} B / B$ is called the Bruhat cell associated to $w \in W$. Here $\dot{w}$ is the representation of $w \in W$ on $G$. For $G=S L(n, \mathbb{C}), W$ is the symmetric group of permutations $\mathcal{S}_{n}$, and $\dot{w}$ is the permutation matrix corresponding to $w \in \mathcal{S}_{n}$. Thus, the Bruhat decomposition partitions flags according to which permutation matrix $\dot{w}$ is needed to perform the factorization $g=n \dot{w} b$ for $g \in G$ with $n \in N$ and $b \in B$. At all values of $t_{k}$ for which the flow $X\left(t_{k}\right)$ is defined, $j_{\Lambda}$ sends $X\left(t_{k}\right)$ into the big cell of the Bruhat decomposition, since $\dot{w}$ is the identity matrix. When the factorization $e^{t_{k} X^{k}(0)}=n\left(t_{k}\right) b\left(t_{k}\right)$ is not possible at time $t_{k}=\bar{t}$, the group element $g=e^{\bar{t} X^{k}(0)}$ can be factored as

$$
e^{\bar{t} X^{k}(0)}=n(\bar{t}) \dot{w} b(\bar{t}),
$$

for some permutation matrix $\dot{w}$. In this case, the flow 3.9) enters the Bruhat cell $N \dot{w} B / B$ at time $t_{k}=\bar{t}$. Ercolani et al. in 21] characterize the Laurent expansion of each pole of $X\left(t_{1}\right)$ in terms of the Bruhat cell that the solution enters at the blow-up time.

The compactification of $\mathcal{M}_{\Lambda}$ where eigenvalues need not be distinct is studied in [70] by a modification of the embedding (3.8) where the Hessenberg matrix $X$ is conjugated to a Jordan matrix $J$ of the spectrum (see [69]). Since the Jordan matrix of $X$ has one block for each eigenvalue, all elements of $\mathcal{M}_{\Lambda}$ are conjugate. Under the Jordan embedding, the maximal torus generated by the flows is diagonal if the eigenvalues are distinct and a product of a diagonal torus and a unipotent group when eigenvalues coincide. Via the Jordan embedding, the $n-1$ flows $X\left(t_{k}\right)=n^{-1}\left(t_{k}\right) X(0) n\left(t_{k}\right)$ in $(\underline{3.6})$, with the appropriate choice of $X(0)$, generate an action of the centralizer of $J$ in $G=S L(n, \mathbb{C})$ on the flag variety $G / B$. As shown in 69 , this group is a semi-direct product of the diagonal torus obtained by setting all the entries above the diagonal equal to zero, and the unipotent group obtained by setting all the diagonal entries equal to 1 . This group action, together with the moment map of the maximal torus, is used in 70 to identify each component in the boundary of the image of $\mathcal{M}_{\Lambda}$ in $G / B$ with a face of the moment polytope.

3.3. The full Kostant-Toda lattice. In this version of the Toda lattice, Ercolani, Flaschka, and Singer [22] connect the expanded phase space with its additional constants of motion introduced by Deift, Li, Nanda and Tomei in [18] (see Section 2.5) and the geometrically enlightening idea of embeddings of the isospectral sets into flag varieties introduced by Ercolani, Flaschka and Haine in [21] (see Section 3.2).

The full Kostant-Toda lattice evolves on the set of full complex Hessenberg matrices

$$
X=\left(\begin{array}{ccccc}
* & 1 & 0 & \cdots & 0 \\
* & * & 1 & \cdots & 0 \\
\vdots & \vdots & \vdots & \ddots & \vdots \\
* & * & * & \cdots & 1 \\
* & * & * & \cdots & *
\end{array}\right)
$$

with arbitrary complex entries below the diagonal. The set of all such $X$ is denoted $\epsilon+\mathfrak{b}_{-}$, where $\epsilon$ is the matrix with 1's on the superdiagonal and zeros elsewhere and $\mathfrak{b}_{-}$is the set of lower triangular complex matrices. Note the decomposition $\mathfrak{s l}_{n}=\mathfrak{b}_{-} \oplus \mathfrak{n}_{+}$where $\mathfrak{n}_{+}$is the set of strictly upper triangular matrices. 
With respect to the symplectic structure on $\epsilon+\mathfrak{b}_{-}$(defined below), the Toda hierarchy (3.5) with $X$ as in (3.13) is completely integrable on the generic leaves. The complete integrability is found in 22] by extending the techniques in [18] to $\epsilon+\mathfrak{b}_{-}$. For complete integrability when $n>3$, we need additional constants of motion independent of the eigenvalues of the initial matrix. These integrals, and the Casimirs (where the flows are trivial) are computed by a chopping construction on $\epsilon+\mathfrak{b}_{-}$ that creates matrices $\phi_{k}(X) \in G L(n-2 k, \mathbb{C})$ for $0 \leq k \leq\left[\frac{(n-1)}{2}\right]$. The coefficients of the polynomial $\operatorname{det}\left(\lambda-\phi_{k}(X)\right)$ are constants of motion referred to as the $k$-chop integrals [22]; they are equivalent to the traces of the powers of $\phi_{k}(X)$. The Hamiltonian system generated by an integral $I(X)$ is

$$
\frac{d}{d t} X=\left[X,(\nabla I(X))_{<0}\right] .
$$

The level sets of the $k$-chop integrals live on the leaves of a symplectic structure on $\epsilon+\mathfrak{b}_{-}$. The symplectic structure can be defined as follows. Write $\mathfrak{s l}_{n}=\mathfrak{n}_{-} \oplus \mathfrak{b}_{+}$where $\mathfrak{n}_{-}$and $\mathfrak{b}_{+}$are the strictly lower triangular and the upper triangular subalgebras. With a nondegenerate inner product $\langle A, B\rangle=\operatorname{tr}(A B)$ on $\mathfrak{s l}_{n}(\mathbb{C})$, we have an isomorphism $\mathfrak{s l}_{n} \cong \mathfrak{s l}_{n}^{*}$, where $\mathfrak{s l}_{n}^{*}=\mathfrak{n}_{-}^{*} \oplus \mathfrak{b}_{+}^{*}=\mathfrak{b}_{+}^{\perp} \oplus \mathfrak{n}_{-}^{\perp}$. With the isomorphisms $\mathfrak{b}_{+}^{*} \cong \mathfrak{n}_{-}^{\perp}=\mathfrak{b}_{-}$and $\mathfrak{n}_{-}^{*} \cong \mathfrak{b}_{+}^{\perp}=\mathfrak{n}_{+}$, we have

$$
\epsilon+\mathfrak{b}_{-} \cong \mathfrak{b}_{+}^{*}
$$

which is the phase space of the full Kostant-Toda lattice. On $\mathfrak{b}_{+}^{*}$, the Lie-Poisson structure is the Kostant-Kirillov form,

$$
\{f, g\}(X)=\left\langle X,\left[\Pi_{\mathfrak{b}+} \nabla f, \Pi_{\mathfrak{b}_{+}} \nabla g\right]\right\rangle \quad \text { for } \quad X \in \mathfrak{b}_{+}^{*},
$$

which stratifies it into symplectic leaves 22 .

Consider the isospectral set $\left(\epsilon+\mathfrak{b}_{-}\right)_{\Lambda}$ with fixed eigenvalues $\Lambda$. Using (3.7), there is a unique lower unipotent matrix $n \in N_{-}$such that for $X \in\left(\epsilon+\mathfrak{b}_{-}\right)_{\Lambda}, X=n C_{\Lambda} n^{-1}$, where $C_{\Lambda}$ is the companion matrix of $X$ :

$$
C_{\Lambda}=\left(\begin{array}{ccccc}
0 & 1 & 0 & \cdots & 0 \\
0 & 0 & 1 & \cdots & 0 \\
\vdots & \vdots & \ddots & \ddots & \vdots \\
0 & 0 & \cdots & 0 & 1 \\
p_{n} & p_{n-1} & \cdots & p_{2} & 0
\end{array}\right)
$$

where the $p_{j}$ 's are the symmetric polynomials of the eigenvalues $\lambda_{j}$, that is,

$$
\operatorname{det}(\lambda I-X)=\prod_{j=1}^{n}\left(\lambda-\lambda_{j}\right)=\lambda^{n}-\sum_{j=2}^{n} p_{j} \lambda^{n-j} .
$$

The mapping

$$
\begin{aligned}
c_{\Lambda}:\left(\epsilon+\mathfrak{b}_{-}\right)_{\Lambda} & \longrightarrow S L(n, \mathbb{C}) / B \\
X & \longmapsto n^{-1} \bmod B
\end{aligned}
$$

is an embedding [51, referred to as the companion embedding. Its image is open and dense in the flag variety. Under this embedding, the $n-1$ flows of the 0 -chop integrals $\frac{1}{k} \operatorname{tr} X^{k}$ generate the action of the centralizer of $C_{\Lambda}$ in $S L(n, \mathbb{C})$ (the group acts by multiplication on the left).

When the eigenvalues $\lambda_{i}$ are distinct, $C_{\Lambda}=V \Lambda V^{-1}$, where $\Lambda=\operatorname{diag}\left(\lambda_{1}, \ldots, \lambda_{n}\right)$ and $V$ is a Vandermonde matrix $V=\left(\lambda_{j}^{i-1}\right)$, and we have

$$
X=n V \Lambda V^{-1} n^{-1} .
$$


This gives an embedding

$$
\begin{aligned}
\Psi_{\Lambda}:\left(\epsilon+\mathfrak{b}_{-}\right)_{\Lambda} & \longrightarrow \quad S L(n, \mathbb{C}) / B \\
X & \longmapsto V^{-1} n^{-1} \bmod B,
\end{aligned}
$$

under which the group generated by Hamiltonian flows of $H_{k}=(1 /(k+1)) \operatorname{tr}\left(X^{k+1}\right)$ for $k=1, \ldots, n-1$ is the maximal diagonal torus. $\Psi_{\Lambda}$ is called the torus embedding.

When the values of the integrals are sufficiently generic (in particular, when the eigenvalues of each $k$-chop are distinct), the flows of the $k$-chop integrals can be organized in the flag variety by the torus embedding as follows [22. (The companion embedding gives a similar structure, but the torus embedding is more convenient since the group action is diagonal.)

Let $S L(m, \mathbb{C}) / P$ denote the quotient of $S L(m, \mathbb{C})$ by the parabolic subgroup $P$ of $S L(m, \mathbb{C})$ whose entries below the diagonal in the first column and to the left of the diagonal in the last row are zero. 22 builds a tower of fibrations where the $k$-chop flows generate a level set of the $(k+1)$-chop integrals in the partial flag variety $S L(n-2 k, \mathbb{C}) / P$ and the $(k+1)$-flows act as a torus action along the fiber, $S L(n-2(k+1), \mathbb{C}) / B$. In the end, the closure of a level set of all the $k$-chop integrals in $S L(n, \mathbb{C}) / B$ is realized as a product of closures of generic torus orbits in the product of partial flag varieties.

$$
S L(n, \mathbb{C}) / P \times S L(n-2, \mathbb{C}) / P \times \cdots \times S L(n-2 M, \mathbb{C}) / P
$$

where $M$ is largest $k$ for which there are $k$-chop integrals.

In 29], Gekhtman and Shapiro generalize the full Kostant-Toda flows and the $k$-chop construction of the integrals to arbitrary simple Lie algebras, showing that the Toda flows on a generic coadjoint orbit in a simple Lie algebra $g$ are completely integrable. A key observation in making this extension is that the 1-chop matrix $\phi_{1}(X)$ can be obtained as the middle $(n-2) \times(n-2)$ block of $A d_{\Gamma(X)}(X)$, where $\Gamma(X)$ is a special element of the Borel subgroup of $G$. This allows one to use the adjoint action of a Borel subgroup, followed by a projection onto a subalgebra, to define the appropriate analog of the 1-chop matrix.

Finally, we note that full Kostant-Toda lattice has a symmetry of order two induced by the nontrivial automorphism of the Dynkin diagram of the Lie algebra $\mathfrak{s l}_{n}(\mathbb{C})$. In terms of the matrices in $\epsilon+\mathfrak{b}_{-}$, the involution is reflection along the anti-diagonal. It is shown by Shipman in [67] that this involution preserves all the $k$-chop integrals and thus defines an involution on each level set of the constants of motion. In the flag variety, the symmetry interchanges the two fixed points of the torus action that correspond to antipodal vertices of the moment polytope under the moment map (3.1).

3.4. Nongeneric full Kostant-Toda flows. When eigenvalues of the initial matrix in $\epsilon+\mathfrak{b}_{-}$ coincide, the torus embedding (3.17) is not defined since each matrix in $\epsilon+\mathfrak{b}_{-}$has one Jordan block for each eigenvalue. In the most degenerate case, when all eigenvalues are zero, [65] uses the companion embedding (3.16) to study the geometry of the flows.

If eigenvalues of each $k$-chop matrix $\phi_{k}(X)$ are distinct but one or more eigenvalues of $\phi_{j}(X)$ and $\phi_{j+1}(X)$ coincide for some $j$, then the torus orbits generated by the $k$-chop integrals in the product (3.18) degenerate into unions of nongeneric orbits [69. This is reflected in splittings of the moment polytopes of the partial flag varieties in (3.18).

From $[69$, let $\mathcal{F}$ be a variety in $S L(n, \mathbb{C}) / P$ defined by fixing the values of the 1-chop integrals $I_{r 1}$, including the Casimir, where the values are chosen so that exactly one eigenvalue, say $\lambda_{i 0}$, of $X$ is also an eigenvalue of $\phi_{1}(X)$. Then $\mathcal{F}$ is the union of the closures of two nongeneric torus orbits such that the images of their closures under the moment map are obtained by splitting the moment polytope of $S L(n, \mathbb{C}) / P$ along an interior face. An example with $n=4$ is illustrated in Figure 3.1. 

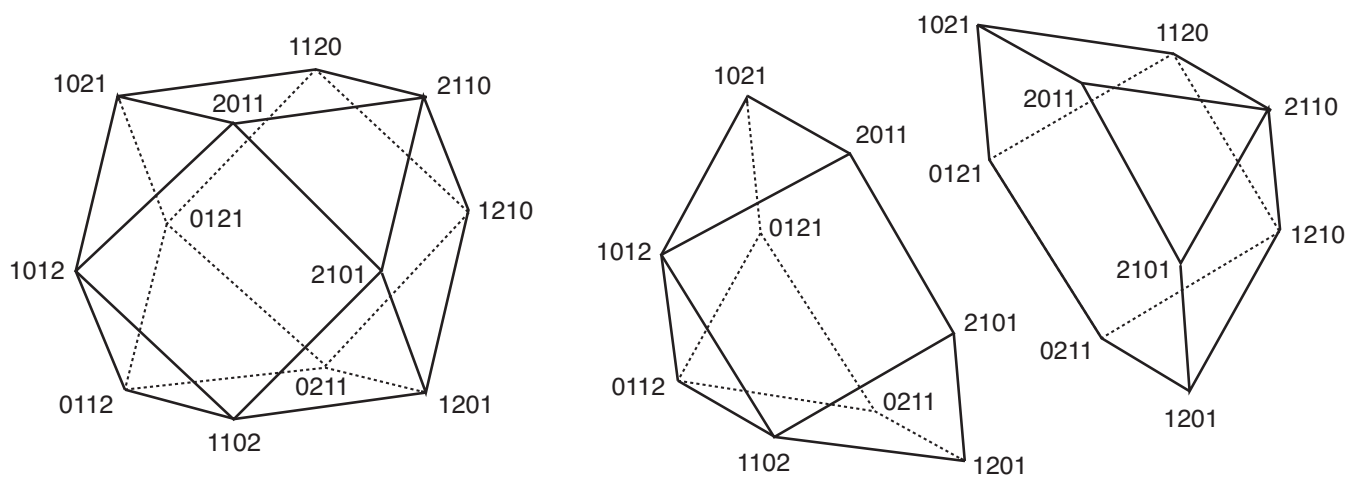

Figure 3.1. Left: the moment polytope of $S L(4, \mathbb{C}) / P$ where the vertices are the weights $\mathrm{L}_{i}-\mathrm{L}_{j}$, e.g., 2110 means $\mathrm{L}_{1}-\mathrm{L}_{4}=2 \mathrm{~L}_{1}+\mathrm{L}_{2}+\mathrm{L}_{3}$. Right: complementary polytopes obtained by splitting the moment polytope of $S L(4, \mathbb{C}) / P$ along an interior hexagon.

This extends to degeneracies in $k$-chop flows for all $k[69$. When a level set of the constants of motion is split into two or more nongeneric torus orbits, separatrices appear in the Toda flows that generate the torus action. The faces along which the polytope is split are the images under the moment map of lower-dimensional torus orbits (the separatrices) that form the interface between the nongeneric orbits of maximum dimension.

For $n=4$, Shipman in 68 determines the monodromy of generic level sets around the singular fibers in the fiber bundle of level sets where the spectrum of the initial matrix is fixed and the single 1-chop integral $I$ is allowed to vary. The flow generated by $I$ produces a $\mathbb{C}^{*}$-bundle with singular fibers over the values of $I$. The singularities occur at two types of coincidences: (1) at values of $I$ where an eigenvalue of the 1-chop matrix coincides with an eigenvalue of the original matrix and (2) at values of $I$ where the two eigenvalues of the 1-chop matrix coincide. In a neighborhood of a singular fiber of the first kind, the monodromy is characterized by a single twist of the noncompact cycle around the cylinder $\mathbb{C}^{*}$. Near a singular fiber of the second kind, the monodromy creates two twists of the noncompact cycle. This double twist appears in the simplest case when $n=2$, around the level set where the two eigenvalues coincide [66].

\section{Other Extensions of the Toda Lattice}

In [47, Kodama and Ye consider an iso-spectral deformation of an arbitrary diagonalizable matrix $L$. The evolution equation is

$$
\frac{d}{d t} L=[P, L] \quad \text { with } \quad P=(L)_{>0}-(L)_{<0} .
$$

The complete integrability of (4.1) is shown in 47] using inverse scattering; it generalizes the method used in [42] to solve the full symmetric real Toda lattice. The method yields an explicit solution to the initial-value problem. The general context of the flow (4.1) includes as special cases the Toda lattices on other classical Lie algebras in addition to $\mathfrak{s l}_{n}(\mathbb{R})$, which is most closely associated with Toda's original system. In this regard, Bogoyavlensky in 8 formulated the Toda lattice on the real split semisimple Lie algebras, which are defined as follows (the formulation below is in the Hessenberg (or Kostant) form, see also [34, 60]): Let $\left\{h_{\alpha_{i}}, e_{ \pm \alpha_{i}}: i=1, \ldots, l\right\}$ be the Chevalley basis of the algebra $\mathfrak{g}$ of rank $l$, that is,

$$
\left[h_{\alpha_{i}}, h_{\alpha_{j}}\right]=0, \quad\left[h_{\alpha_{i}}, e_{ \pm \alpha_{j}}\right]= \pm C_{j i} e_{ \pm \alpha_{j}}, \quad\left[e_{\alpha_{i}}, e_{-\alpha_{j}}\right]=\delta_{i j} h_{\alpha_{j}},
$$


where $\left(C_{i j}\right)_{1 \leq i, j \leq l}$ is the Cartan matrix and $C_{i j}=\alpha_{i}\left(h_{\alpha_{j}}\right)$. Then the (nonperiodic) Toda lattice associated with the Lie algebra $g$ is governed by the Lax equation

$$
\frac{d L}{d t}=[A, L]
$$

where $L$ is a Jacobi element of $\mathfrak{g}$ and $A$ is the projection of $L$ onto $\mathfrak{n}_{-}$, as

$$
\left\{\begin{array}{l}
L(t)=\sum_{i=1}^{l} f_{i}(t) h_{\alpha_{i}}+\sum_{i=1}^{l}\left(g_{i}(t) e_{-\alpha_{i}}+e_{\alpha_{i}}\right), \\
A(t)=-\Pi_{\mathfrak{n}_{-}} L(t)=-\sum_{i=1}^{l} g_{i}(t) e_{-\alpha_{i}} .
\end{array}\right.
$$

The complete integrability is based on the existence of the Chevalley invariants of the algebra, and the geometry of the isospectral variety has been discussed in terms of the representation theory of Lie groups by Kostant in 52 for the cases where $g_{i}$ are real positive, or complex. The general case for real $g_{i}$ 's is studied by Casian and Kodama [10, 11, which extends the results in the $\mathfrak{s l}_{n}(\mathbb{R})$ Toda lattice in the Hessenberg form (see Section 2.4) to the Toda lattice for any real split semisimple Lie algebra.

The Lax equation (4.2) then gives

$$
\frac{d f_{i}}{d t}=g_{i}, \quad \text { and } \quad \frac{d g_{i}}{d t}=-\left(\sum_{j=1}^{l} C_{i j} f_{j}\right) g_{i}
$$

from which the $\tau$-functions are defined as

$$
f_{i}(t)=\frac{d}{d t} \ln \tau_{i}(t), \quad g_{i}(t)=g_{i}(0) \prod_{j=1}^{l}\left(\tau_{j}(t)\right)^{-C_{i j}} .
$$

In the case of $\mathfrak{g}=\mathfrak{s l}_{n}(\mathbb{R})$, those equations are (2.22). Note here that the superdiagonal of $L(t)$ is $\operatorname{diag}\left(f_{1}-f_{2}, f_{2}-f_{3}, \ldots, f_{l}-f_{l+1}\right)$ with $n=l+1$. Those extensions have been discussed by many authors (see for example [34, 60]). One should note that Bogoyavlensky in [8] also formulates those Toda lattices for affine Kac-Moody Lie algebras, and they give the periodic Toda lattice. There has been much progress in understanding these periodic Toda lattices, but we will not cover the subject in this paper (see, for example [2, 3, 20, 62, 63]).

From the viewpoint of Lie theory, the underlying structure of the integrable systems is based on the Lie algebra splitting, e.g. $\mathfrak{s l}_{n}=\mathfrak{b}_{-} \oplus \mathfrak{s o}_{n}$ (the QR-factorization) for the symmetric Toda lattice, and $\mathfrak{s l}_{n}=\mathfrak{b}_{+} \oplus \mathfrak{n}_{-}$(the LU decomposition) for the Hessenberg form of Toda lattice. Then one can also consider the following form of the evolution equation,

$$
\frac{d}{d t} L=[Q, L] \quad \text { with } \quad Q=\Pi_{\mathfrak{g}_{1}}(L),
$$

where $\mathfrak{g}_{1}$ is a subalgebra in the Lie algebra splitting $\mathfrak{s l}_{n}=\mathfrak{g}_{1} \oplus \mathfrak{g}_{2}$. In this regard, we mention here the following two interesting systems directly connecting to the Toda lattice:

4.1. The Kac-van Moerbeke system. [37]: We take $\mathfrak{g}_{1}=\mathfrak{s o}_{2 n}$, and consider the equation for $L \in \mathfrak{s o}_{2 n}$. Since $L^{2 k-1} \in \mathfrak{s o}_{2 n}$, the even flows are all trivial. Let $L$ be given by a tridiagonal form,

$$
L=\left(\begin{array}{ccccc}
0 & \alpha_{1} & 0 & \cdots & 0 \\
-\alpha_{1} & 0 & \alpha_{2} & \cdots & 0 \\
\vdots & \vdots & \ddots & \cdots & \cdots \\
0 & 0 & \cdots & 0 & \alpha_{2 n-1} \\
0 & 0 & \cdots & -\alpha_{2 n-1} & 0
\end{array}\right) \in \mathfrak{s o}_{2 n}(\mathbb{R})
$$


Then the even flows are the Kac-van Moerbeke hierarchy, $\frac{\partial L}{\partial t_{2 j}}=\left[\Pi_{\mathfrak{s o}}\left(L^{2 j}\right), L\right]$, where the first member of $t_{2}$-flow gives

$$
\frac{\partial \alpha_{k}}{\partial t_{2}}=\alpha_{k}\left(\alpha_{k-1}^{2}-\alpha_{k+1}^{2}\right), \quad k=1, \ldots, 2 n-1,
$$

with $\alpha_{0}=\alpha_{2 n}=0$. This system is equivalent to the symmetric Toda lattice which can be written as (4.5) for the square $L^{2}$. Note here that $L^{2}$ is a symmetric matrix given by

$$
L^{2}=T^{(1)} \otimes\left(\begin{array}{ll}
1 & 0 \\
0 & 0
\end{array}\right)+T^{(2)} \otimes\left(\begin{array}{ll}
0 & 0 \\
0 & 1
\end{array}\right),
$$

where $T^{(i)}$, for $i=1,2$, are $n \times n$ symmetric tridiagonal matrices given by

$$
T^{(i)}=\left(\begin{array}{ccccc}
b_{1}^{(i)} & a_{1}^{(i)} & 0 & \cdots & 0 \\
a_{1}^{(i)} & b_{2}^{(i)} & a_{2}^{(i)} & \cdots & 0 \\
\vdots & \vdots & \ddots & \ddots & \vdots \\
0 & 0 & \cdots & b_{n-1}^{(i)} & a_{n-1}^{(i)} \\
0 & 0 & \cdots & a_{n-1}^{(i)} & b_{n}^{(i)}
\end{array}\right),
$$

with $a_{k}^{(1)}=\alpha_{2 k-1} \alpha_{2 k}, b_{k}^{(1)}=-\alpha_{2 k-2}^{2}-\alpha_{2 k-1}^{2}, a_{k}^{(2)}=\alpha_{2 k} \alpha_{2 k+1}$, and $b_{k}^{(2)}=-\alpha_{2 k-1}^{2}-\alpha_{2 k}^{2}$ (see [33]). Then one can show that each $T^{(i)}$ gives the symmetric Toda lattice, that is, the Kac-van Moerbeke hierarchy for $L^{2}$ matrix splits into two Toda lattices,

$$
\frac{\partial T^{(i)}}{\partial t_{2 j}}=\left[\Pi_{s o}\left(T^{(i)}\right)^{j}, T^{(i)}\right] \quad \text { for } \quad i=1,2 .
$$

The equations for $T^{(i)}$ are connected by the Miura-type transformation, with the functions $\left(a_{k}^{(i)}, b_{k}^{(i)}\right)$, through the Kac-van Moerbeke variables $\alpha_{k}$ (see [33]).

4.2. The Pfaff lattice for a symplectic matrix. [1, 43, 44]: The Pfaff lattice is defined in the same form with $\mathfrak{g}_{1}=\mathfrak{s p}_{2 n}$ and $L$ in the Hessenberg form with $2 \times 2$ block structure. In particular, we consider the case $L \in \mathfrak{s p}_{2 n}$ having the form,

$$
L=\left(\begin{array}{cc|cc|c|c}
0 & \sigma_{1} & 0 & 0 & \cdots & 0_{2} \\
b_{1} & 0 & a_{1} & 0 & \cdots & \\
\hline 0 & 0 & 0 & \sigma_{2} & \cdots & 0_{2} \\
a_{1} & 0 & b_{2} & 0 & & \vdots \\
\hline \vdots & \vdots & \ddots & \vdots \\
\hline 0_{2} & \multicolumn{2}{|c|}{0_{2}} & \cdots & \begin{array}{cc}
0 & \sigma_{n} \\
b_{n} & 0
\end{array}
\end{array}\right) \in \mathfrak{s p}_{2 n}(\mathbb{R})
$$

where $0_{2}$ is the $2 \times 2$ zero matrix. The variables $\left(a_{k}, b_{k}\right)$ and $\sigma_{k}= \pm 1$ are those in the indefinite Toda lattice in (2.15) through $f_{k}=\sigma_{k} b_{k}$ and $g_{k}=\sigma_{k} \sigma_{K=1} a_{k}^{2}$. It should be noted again that the odd members are trivial (since $L^{2 k-1} \in \mathfrak{s p}_{2 n}$ ), and the even members give the indefinite Toda lattice hierarchy [44]. Here one should note that $L^{2}$ can be written as

$$
L^{2}=\tilde{L}^{T} \otimes\left(\begin{array}{ll}
1 & 0 \\
0 & 0
\end{array}\right)+\tilde{L} \otimes\left(\begin{array}{ll}
0 & 0 \\
0 & 1
\end{array}\right)
$$


where $\tilde{L}=\tilde{D}^{-1} X \tilde{D}$ with $\tilde{D}=\operatorname{diag}\left(1, \sigma_{2} a_{1}, \ldots, \sigma_{n} a_{n-1}\right)$. Then one can show that the generator $Q_{2 j}$ of the Lax equation is given by

$$
Q_{2 j}=\Pi_{\mathfrak{s p}}\left(L^{2 j}\right)=-\tilde{B}_{j}^{T} \otimes\left(\begin{array}{ll}
1 & 0 \\
0 & 0
\end{array}\right)+\tilde{B}_{j} \otimes\left(\begin{array}{cc}
0 & 0 \\
0 & 1
\end{array}\right),
$$

where $\tilde{B}_{j}=\frac{1}{2}\left[\left(\tilde{L}^{j}\right)_{>0}-\left(\tilde{L}^{j}\right)_{<0}\right]$. Then the hierarchy $\frac{d}{d t} L=\left[Q_{2 j}, L\right]$ gives the indefinite Toda lattice hierarchy (see Section 2.4 and $[45,46]$ ).

\section{The full Kostant-Toda lattice in Real variables}

Here we consider the full Kostant-Toda hierarchy (3.5) in real variables, where we write

$$
\frac{\partial X}{\partial t_{k}}=\left[\left(X^{k}\right)_{\geq 0}, X\right] \quad \text { for } \quad k=1,2, \ldots, n-1 .
$$

We let $\mathbf{t}:=\left(t_{1}, \ldots, t_{n-1}\right)$ denote the multi-time variables for the flows in the hierarchy. As is the case of the complex full Kostant-Toda lattice, the solution space can be described by the flag variety $G / B$. Here we consider the asymptotic behavior of the solutions for the regular flows of the full Kostant-Toda hierarchy. Those regular solutions are associated to points in the totally nonnegative (tnn) flag variety, denoted by $(G / B)_{\geq 0}$. Then we discuss the moment map images of the regular flows of the full Kostant-Toda hierarchy. This section is a brief review of [50 which provides a geometric structure of the iso-spectral variety for the full Kostant-Toda flows including nongeneric cases.

5.1. Totally nonnegative parts of flag varieties. We begin with a brief review of the tnn parts of the flag variety, $(G / B)_{\geq 0}$ where $G=S L(n, \mathbb{R})$.

For each $1 \leq i \leq n-1$ we have a homomorphism $\phi_{i}: S L(2, \mathbb{R}) \rightarrow S L(n, \mathbb{R})$ such that

$$
\phi_{i}\left(\begin{array}{ll}
a & b \\
c & d
\end{array}\right)=\left(\begin{array}{cccccc}
1 & & & & & \\
& \ddots & & & & \\
& & a & b & & \\
& & c & d & & \\
& & & & \ddots & \\
& & & & & 1
\end{array}\right) \in S L(n, \mathbb{R}),
$$

that is, $\phi_{i}$ replaces a $2 \times 2$ block of the identity matrix with $\left(\begin{array}{ll}a & b \\ c & d\end{array}\right)$, where $a$ is at the $(i, i)$-entry. We have 1-parameter subgroups of $G$ defined by

$$
x_{i}(m)=\phi_{i}\left(\begin{array}{cc}
1 & m \\
0 & 1
\end{array}\right) \text { and } y_{i}(m)=\phi_{i}\left(\begin{array}{cc}
1 & 0 \\
m & 1
\end{array}\right) \text {, where } m \in \mathbb{R} \text {. }
$$

The simple reflections $s_{i} \in W=\mathcal{S}_{n}$ are given by $s_{i}:=\dot{s}_{i} T$ where $\dot{s_{i}}:=\phi_{i}\left(\begin{array}{cc}0 & -1 \\ 1 & 0\end{array}\right)$, and any $w \in W$ can be expressed as a product $w=s_{i_{1}} s_{i_{2}} \ldots s_{i_{\ell}}$ with $\ell=\ell(w)$ factors. Here $\ell(w)$ denotes the length of $w$. We set $\dot{w}=\dot{s}_{i_{1}} \dot{s}_{i_{2}} \ldots \dot{s}_{i_{\ell}}$.

There are two opposite Bruhat decompositions of $G / B$ :

$$
G / B=\bigsqcup_{w \in W} B \dot{w} B / B=\bigsqcup_{v \in W} N \dot{v} B / B .
$$

We define the intersection of opposite Bruhat cells

$$
\mathcal{R}_{v, w}:=(B \dot{w} B / B) \cap(N \dot{v} B / B),
$$


which is nonempty precisely when $v \leq w$. The strata $\mathcal{R}_{v, w}$ are often called Richardson varieties.

Now we define the totally nonnegative part of the flag.

Definition 5.1. [55] The tnn part $N_{\geq 0}$ of $N$ is defined to be the semigroup in $N$ generated by the $y_{i}(p)$ for $p \in \mathbb{R}_{\geq 0}$ in (5.2). The tnn part $(G / B)_{\geq 0}$ of $G / B$ is defined by

$$
(G / B)_{\geq 0}:=\overline{\left\{n B \mid n \in N_{\geq 0}\right\}}
$$

where the closure is taken inside $G / B$ in its real topology. We sometimes refer to $(G / B)_{\geq 0}$ as the tnn flag variety.

Lusztig [55, 54] introduced a natural decomposition of $(G / B)_{\geq 0}$ : For $v, w \in W$ with $v \leq w$, let

$$
\mathcal{R}_{v, w}^{>0}:=\mathcal{R}_{v, w} \cap(G / B)_{\geq 0} .
$$

Then the tnn part of the flag variety $G / B$ has the decomposition,

$$
(G / B)_{\geq 0}=\bigsqcup_{w \in W}\left(\bigsqcup_{v \leq w} \mathcal{R}_{v, w}^{>0}\right) .
$$

Let $\mathbf{w}:=s_{i_{1}} \ldots s_{i_{m}}$ be a reduced expression for $w \in W$. A subexpression $\mathbf{v}$ of $\mathbf{w}$ is a word obtained from the reduced expression $\mathbf{w}$ by replacing some of the factors with 1 . For example, consider a reduced expression in the symmetric group $\mathcal{S}_{4}$, say $s_{3} s_{2} s_{1} s_{3} s_{2} s_{3}$. Then $1 s_{2} 11 s_{2} s_{3}$ is a subexpression of $s_{3} s_{2} s_{1} s_{3} s_{2} s_{3}$. Given a subexpression $\mathbf{v}$, we set $v_{(k)}$ to be the product of the leftmost $k$ factors of $\mathbf{v}$, if $k \geq 1$, and $v_{(0)}=1$. To parametrize each component of $\mathcal{R}_{v, w}^{>0}$, we need the following definition of the subexpressions of $w$ :

Definition 5.2. [19, 57, Given a subexpression $\mathbf{v}$ of $\mathbf{w}=s_{i_{1}} s_{i_{2}} \ldots s_{i_{m}}$, we define

$$
\begin{aligned}
J_{\mathbf{v}}^{\circ} & :=\left\{k \in\{1, \ldots, m\} \mid v_{(k-1)}<v_{(k)}\right\}, \\
J_{\mathbf{v}}^{+} & :=\left\{k \in\{1, \ldots, m\} \mid v_{(k-1)}=v_{(k)}\right\}, \\
J_{\mathbf{v}}^{\bullet} & :=\left\{k \in\{1, \ldots, m\} \mid v_{(k-1)}>v_{(k)}\right\} .
\end{aligned}
$$

The subexpression $\mathbf{v}$ is called nondecreasing if $v_{(j-1)} \leq v_{(j)}$ for all $j=1, \ldots, m$, e.g. if $J_{\mathbf{v}}^{\bullet}=\emptyset$. It is called distinguished if we have $v_{(j)} \leq v_{(j-1)} s_{i_{j}}$ for all $j \in\{1, \ldots, m\}$. In other words, if right multiplication by $s_{i_{j}}$ decreases the length of $v_{(j-1)}$, then in a distinguished subexpression we must have $v_{(j)}=v_{(j-1)} s_{i_{j}}$. Finally, $\mathbf{v}$ is called a positive distinguished subexpression (or a PDS for short) if $v_{(j-1)}<v_{(j-1)} s_{i_{j}}$ for all $j \in\{1, \ldots, m\}$. In other words, it is distinguished and nondecreasing.

It is then quite important to note that given $v \leq w$ and a reduced expression $\mathbf{w}$ for $w$, there is a unique PDS $\mathbf{v}_{+}$for $v$ contained in $\mathbf{w}[57,49$. The following theorem then provides a parameterization of the tnn part of the flag variety.

Theorem 5.3. [57, Proposition 5.2, Theorem 11.3] Choose a reduced expression $\mathbf{w}=s_{i_{1}} \ldots s_{i_{m}}$ for $w$ with $\ell(w)=m$. To $v \leq w$ we associate the unique $P D S \mathbf{v}_{+}$for $v$ in $\mathbf{w}$. Then $J_{\mathbf{v}^{+}}^{\bullet}=\emptyset$. We define

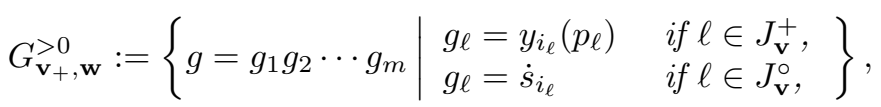

where each $p_{\ell}$ ranges over $\mathbb{R}_{>0}$. The set $G_{\mathbf{v}_{+}, \mathbf{w}}^{>0}$ lies in $N \dot{v} \cap B \dot{w} B, G_{\mathbf{v}_{+}, \mathbf{w}}^{>0} \cong \mathbb{R}_{>0}^{\ell(w)-\ell(v)}$, and the map $g \mapsto g B$ defines an isomorphism

$$
G_{\mathbf{v}_{+}, \mathbf{w}}^{>0} \stackrel{\sim}{\longrightarrow} \mathcal{R}_{v, w}^{>0}
$$


5.1.1. The Grassmannian and its tnn part. The real Grassmannian $G r(k, n)$ is the space of all $k$ dimensional subspaces of $\mathbb{R}^{n}$. An element of $\operatorname{Gr}(k, n)$ can be viewed as a full-rank $k \times n$ matrix $A$ modulo left multiplication by nonsingular $k \times k$ matrices. In other words, two $k \times n$ matrices are equivalent, i.e. they represent the same point in $\operatorname{Gr}(k, n)$, if and only if they can be obtained from each other by row operations.

Let $\left(\begin{array}{c}{[n]} \\ k\end{array}\right)$ be the set of all $k$-element subsets of $[n]:=\{1, \ldots, n\}$. For $I \in\left(\begin{array}{c}{[n]} \\ k\end{array}\right)$, let $\Delta_{I}(A)$ be the Plücker coordinate, that is, the maximal minor of the $k \times n$ matrix $A$ located in the column set $I$. The map $A \mapsto\left(\Delta_{I}(A)\right)$, where $I$ ranges over $\left(\begin{array}{c}{[n]} \\ k\end{array}\right)$, induces the Plücker embedding $\operatorname{Gr}(k, n) \hookrightarrow \mathbb{R P}\left(\begin{array}{l}n \\ k\end{array}\right)-1$.

Just as for the flag variety, one may identify the Grassmannian with a homogeneous space. Let $P_{k}$ be the parabolic subgroup which fixes the $k$-dimensional subspace spanned by $e_{1}, \ldots, e_{k}$. (This is a block upper-triangular matrix containing $B$.) Then we may identify $G r(k, n)$ with the space of cosets $G / P_{k}$.

There is a natural projection $\pi_{k}: G / B \rightarrow G r(k, n)$. One may equivalently express this projection as the map $\pi_{k}: G / B \rightarrow G / P_{k}$, where $\pi_{k}(g B)=g P_{k}$. Abusing notation, we simply write $\pi_{k}(g)=A_{k}$ with $A_{k} \in G r(k, n) \cong G / P_{k}$ instead of $\pi_{k}(g B)=g P_{k}$.

Concretely, for $g \in G, \pi_{k}(g)$ is represented by the $k \times n$ matrix $A_{k}$ consisting of the leftmost $k$ columns of $g$, i.e.

$$
g=\left(\begin{array}{ccccc}
g_{1,1} & \cdots & g_{1, k} & \cdots & g_{1, n} \\
\vdots & \ddots & \vdots & \vdots & \vdots \\
g_{k, 1} & \cdots & g_{k, k} & \cdots & g_{k, n} \\
\vdots & \vdots & \vdots & \vdots & \vdots \\
g_{n, 1} & \cdots & g_{n, k} & \cdots & g_{n, n}
\end{array}\right) \quad \longmapsto \quad A_{k}=\left(\begin{array}{ccccc}
g_{1,1} & \cdots & g_{k, 1} & \cdots & g_{n, 1} \\
\vdots & \ddots & \vdots & \vdots & \vdots \\
g_{1, k} & \cdots & g_{k, k} & \cdots & g_{n, k}
\end{array}\right) .
$$

This is equivalent to the following formula using the Plücker embedding into the projectivization of the wedge product space $\mathbb{P}\left(\bigwedge^{k} \mathbb{R}^{n}\right) \cong \mathbb{R P}\left(\begin{array}{c}n \\ k\end{array}\right)-1$ with the standard basis $\left\{e_{i}: i=1, \ldots, n\right\}$,

$$
g \cdot e_{1} \wedge \cdots \wedge e_{k}=\sum_{1 \leq i_{1}<\cdots<i_{k} \leq n} \Delta_{i_{1}, \ldots, i_{k}}\left(A_{k}\right) e_{i_{1}} \wedge \cdots \wedge e_{i_{k}}
$$

The Plücker coordinates $\Delta_{i_{1}, \ldots, i_{k}}\left(A_{k}\right)$ are then given by

$$
\Delta_{i_{1}, \ldots, i_{k}}\left(A_{k}\right)=\left\langle e_{i_{1}} \wedge \cdots \wedge e_{i_{k}}, g \cdot e_{1} \wedge \cdots \wedge e_{k}\right\rangle
$$

where $\langle\cdot, \cdot\rangle$ is the usual inner product on $\bigwedge^{k} \mathbb{R}^{n}$.

Now the tnn part of the Grassmannian is then defined as follows:

Definition 5.4. The tnn part of the Grassmannian $G r(k, n)_{\geq 0}$ is the image $\pi_{k}\left((G / B)_{\geq 0}\right)$. Equivalently, $\operatorname{Gr}(k, n)_{\geq 0}$ is the subset of $\operatorname{Gr}(k, n)$ such that all Plücker coordinates are nonnegative.

Let $W_{k}=\left\langle s_{1}, \ldots, \hat{s}_{k}, \ldots, s_{n-1}\right\rangle$ be a parabolic subgroup of $W=\mathcal{S}_{n}$ obtained by deleting the transposition $s_{k}$ from the generating set. Let $W^{k}$ denote the set of minimal-length coset representatives of $W / W_{k}$. Recall that a descent of a permutation $z$ is a position $j$ such that $z(j)>z(j+1)$. Then $W^{k}$ is the subset of permutations which have at most one descent, and if it exists, that descent must be in position $k$.

Rietsch in 64 shows that the tnn part of the Grassmannian $\operatorname{Gr}(k, n)_{\geq 0}$ has a cellular decomposition (cf. (5.3) $)$,

$$
G r(k, n)_{\geq 0}=\bigsqcup_{w \in W^{k}} \bigsqcup_{v \leq w} \mathcal{P}_{v, w}^{>0}
$$

where $\mathcal{P}_{v, w}^{>0}=\pi_{k}\left(\mathcal{R}_{v, w}^{>0}\right)$ 
Definition 5.5. Let $M$ be an $n \times n$ matrix with real entries. Any determinant of a $k \times k$ submatrix (for $1 \leq k \leq n$ ) is called a flag minor if its set of columns is precisely $\{1,2, \ldots, k\}$, the leftmost $k$ columns of $M$. Let $\Delta_{I_{k}}^{k}(M)$ denote the flag minor where $I_{k}=\left\{i_{1}, \ldots, i_{k}\right\}$ is the set of rows. And we say that $M$ is flag nonnegative if all of its flag minors are nonnegative.

Note that the flag minors of $g \in G$ are precisely the Plücker coordinates of the projections of $g B$ to the various Grassmannians $\pi_{k}(g B)$ for $1 \leq k \leq n$. Then, one can show [50] that any $g \in G_{\mathbf{v}_{+}, \mathbf{w}}^{>0}$ is a flag nonnegative. That is, the Plücker coordinates in (5.6) are all nonnegative when $A_{k}$ is given by the matrix $g \in G_{\mathbf{v}+, \mathbf{w}}^{>0}$.

For any $z \in W$ we define the ordered set $z \cdot[k]=\{z(1), \ldots, z(k)\}$. (By ordered set, we mean that we sort the elements of $z \cdot[k]$ according to their value.) Then we have the following.

Lemma 5.6. [50] Let $v \leq w$ be elements in $W=\mathcal{S}_{n}$, and choose $z \in \mathcal{S}_{n}$ arbitrarily. Choose a reduced subexpression $\mathbf{w}$ for $w$; this determines the $P D S \mathbf{v}_{+}$for $v$ in $\mathbf{w}$. Choose any $g \in G_{\mathbf{v}_{+}, \mathbf{w}}^{>0}$. Then we have

$$
\Delta_{z \cdot[k]}^{k}(g)>0 \quad \text { for } \quad 1 \leq k \leq n
$$

if and only if

$$
v \leq z \leq w
$$

This lemma is a key to determine the polytope structure of nongeneric flows of the full KostantToda hierarchy.

5.2. Full Kostant-Toda flows with totally nonnegative initial data. Let us recall that the solution of the full Kostant-Toda can be found by the companion embedding (3.16) with the factorization $\exp \left(\Theta_{X^{\mathbf{0}}}(\mathbf{t})\right)=n(\mathbf{t}) b(\mathbf{t})$ where $\Theta_{X^{\mathbf{0}}}(\mathbf{t}):=\sum_{j=1}^{n-1}\left(X^{\mathbf{0}}\right)^{j} t_{j}$ with the initial matrix $X^{\mathbf{0}}=X(\mathbf{0})$. Then we have the solution $X(\mathbf{t})=n^{-1}(\mathbf{t}) X^{\mathbf{0}} n(\mathbf{t})$. This can be stated in the following diagram [28, 10, 50]:

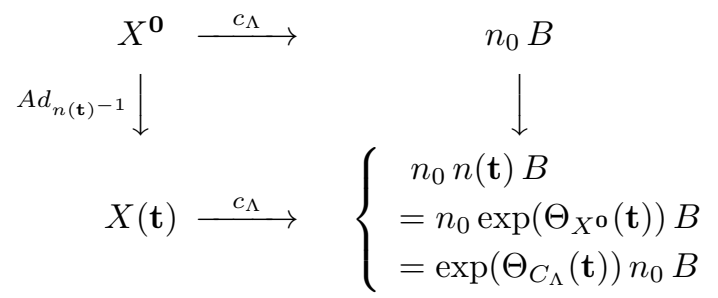

where $X^{\mathbf{0}}=n_{0}^{-1} C_{\Lambda} n_{0}$. That is, the initial matrix $X^{\mathbf{0}}=X(\mathbf{0})$ determines the element $n_{0} \in N$, and each full Kostant-Toda flow corresponds to an $\exp \left(\Theta_{C_{\Lambda}}(\mathbf{t})\right)$-orbit on the flag variety with the initial point $n_{0} B$.

We now associate to each matrix $g \in G_{\mathbf{v}_{+}, \mathbf{w}}^{>0}$ (representing a point of $\mathcal{R}_{v, w}^{>0}$ ) an initial matrix $X^{\mathbf{0}}$ for the full Kostant-Toda hierarchy. First we note that the $\tau$-functions for the hierarchy can be also found in the same form as in (2.21), i.e.

$$
\tau_{k}(\mathbf{t})=\left[\exp \left(\Theta_{X^{0}}(\mathbf{t})\right)\right]_{k} \quad \text { for } \quad k=1, \ldots, n-1 .
$$

We then express the $\tau$-functions with the initial matrix $X^{0}$ in terms of $g$.

Recall that $C_{\Lambda} V=V \Lambda$ where $V$ is the Vandermonde matrix $V=\left(\lambda_{j}^{i-1}\right)$, and that $[M]_{k}$ denotes the $k$ th principal minor of the matrix $M$. Then, for each matrix $g \in G_{\mathbf{v}_{+}, \mathbf{w}}^{>0}$ we can associate an initial matrix $X^{\mathbf{0}} \in \mathcal{F}_{\Lambda}$, defined by $X^{\mathbf{0}}=n_{0}^{-1} C_{\Lambda} n_{0}$, where $n_{0} \in N$ and $b_{0} \in B$ are uniquely determined 
by the equation $E g=n_{0} b_{0}$ (this decomposition is true when $g \in G_{\mathbf{v}_{+}, \mathbf{w}}^{>0}$ ). Then, the $\tau$-functions for the full Kostant-Toda hierarchy with initial matrix $X^{\mathbf{0}}$ are given by

$$
\tau_{k}(\mathbf{t})=\left[\exp \left(\Theta_{C_{\Lambda}}(\mathbf{t})\right) n_{0}\right]_{k}=d_{k}\left[V \exp \left(\Theta_{\Lambda}(\mathbf{t})\right) g\right]_{k}
$$

where $d_{k}=\left[b_{0}^{-1}\right]_{k}$.

Remark 5.7. The formula $\exp \left(\Theta_{C_{\Lambda}}(\mathbf{t})\right) n_{0}=E \exp \left(\Theta_{\Lambda}(\mathbf{t})\right) g b_{0}^{-1}$ implies that the full Kostant-Toda flow gives a (noncompact) torus action on the flag variety. More precisely, the torus $\left(\mathbb{R}_{>0}\right)^{n}$ acts by $\exp \left(\Theta_{\Lambda}(\mathbf{t})\right)$ on the basis vectors consisting of the columns of the Vandermonde matrix $V$, that is, we have $\exp \left(\Theta_{X^{\mathbf{o}}}(\mathbf{t})\right) n_{0} B=V \exp \left(\Theta_{\Lambda}(\mathbf{t})\right) g B$.

Then using the Binet-Cauchy lemma to (5.10) and $A_{k}=\pi_{k}(g)$, the $\tau$-function can be written as

$$
\tau_{k}(\mathbf{t})=d_{k} \sum_{I \in\left(\begin{array}{c}
{[n]} \\
k
\end{array}\right)} \Delta_{I}\left(A_{k}\right) E_{I}(\mathbf{t})
$$

where $E_{I}(\mathbf{t})$ for $I=\left\{i_{1}, \ldots, i_{k}\right\}$ is defined by

$$
E_{I}(\mathbf{t}):=\prod_{\ell<m}\left(\lambda_{i_{m}}-\lambda_{i_{\ell}}\right) \prod_{j \in I}^{n} E_{j}(\mathbf{t}) \quad \text { with } \quad E_{j}(\mathbf{t})=e^{\theta_{j}(\mathbf{t})} .
$$

Since $g \in G_{\mathbf{v}_{+}, \mathbf{w}}^{>0}$ is a flag nonnegative, i.e. $\Delta_{I_{k}}\left(A_{k}\right) \geq 0$ for all $I_{k} \in\left(\begin{array}{c}{[n]} \\ k\end{array}\right)$, the $\tau$-function is signdefinite. This implies that the full Kostant-Toda flow is complete for all $\mathbf{t}=\left(t_{1}, \ldots, t_{n-1}\right) \in \mathbb{R}^{n-1}$, when the initial matrix $X^{0}$ comes from a point in $G_{\mathbf{v}_{+}, \mathbf{w}}^{>0}$.

Remark 5.8. The $\tau$-function in (5.11) has the Wronskian structure, that is, if we define the functions $\left\{f_{1}, \ldots, f_{k}\right\}$ by

then we have

$$
\left(f_{1}(\mathbf{t}), \ldots, f_{k}(\mathbf{t})\right):=\left(E_{1}(\mathbf{t}), \ldots, E_{n}(\mathbf{t})\right) A_{k}^{T}
$$

$$
\tau_{k}(\mathbf{t})=d_{k} \operatorname{Wr}\left(f_{1}(\mathbf{t}), \ldots, f_{k}(\mathbf{t})\right)
$$

where the Wronskian is for the $t_{1}$-variable. Furthermore, if we identify the first three variables as $t_{1}=x, t_{2}=y$ and $t_{3}=t$ in (5.11), then we obtain the $\tau$-function for the KP equation [38] which gives rise to soliton solutions of the KP equation from the Grassmannian $G r(k, n)$ [4] (see [41] for a review of the KP solitons). That is, $\tau_{k}$ is associated with a point of the Grassmannian $\operatorname{Gr}(k, n)$. Then the set of $\tau$-functions $\left(\tau_{1}, \ldots, \tau_{n-1}\right)$ is associated with a point of the flag variety, and the solution space of the full Kostant-Toda hierarchy is naturally given by the complete flag variety.

5.3. Asymptotic behavior of the full Kostant-Toda lattice. Here we consider the asymptotics of the solution $X(\mathbf{t})$ to the full Kostant-Toda lattice where $X(\mathbf{0})=X^{\mathbf{0}}$ is the initial matrix associated with $g \in G_{\mathbf{v}_{+}, \mathbf{w}}^{>0}$, i.e. the tnn part of the flag variety.

Recall that we have a fixed order $\lambda_{1}<\cdots<\lambda_{n}$ on the eigenvalues, and that $z \cdot[k]$ denotes the ordered set $\{z(1), z(2), \ldots, z(k)\}$. Since $A_{k}=\pi_{k}(g)$ and $g \in N \dot{v} \cap B \dot{w} B$ (by Theorem [5.3), the lexicographically maximal and minimal elements in $\mathcal{M}\left(A_{k}\right)$ are respectively given by $w \cdot[k]$ and $v \cdot[k]$. Because of the order $\lambda_{1}<\cdots<\lambda_{n}$, we have the following with $E_{i}(t)=e^{\theta_{i}(t)}$ in (5.12),

$$
\begin{aligned}
& E_{1} \ll E_{2} \ll \cdots \ll E_{n}, \quad \text { as } \quad t \rightarrow \infty, \\
& E_{1} \gg E_{2} \gg \cdots \gg E_{n}, \quad \text { as } \quad t \rightarrow-\infty,
\end{aligned}
$$

This implies that each $\tau_{k}(t)$-function from (5.11) has the following asymptotic behavior:

$$
\tau_{k}(t) \longrightarrow \begin{cases}E_{w \cdot[k]}(t) & \text { as } t \rightarrow \infty \\ E_{v \cdot[k]}(t) & \text { as } \quad t \rightarrow-\infty\end{cases}
$$


Then the diagonal element $f_{k}(t)$ in $X(t)$ in the form (2.22) can be calculated as

$$
f_{k}(t)=\frac{d}{d t} \ln \frac{\tau_{k}}{\tau_{k-1}} \longrightarrow \frac{d}{d t} \ln \frac{E_{w \cdot[k]}}{E_{w \cdot[k-1]}}=\frac{d}{d t} \ln E_{w(k)}=\lambda_{w(k)} \quad \text { as } t \rightarrow \infty .
$$

This implies that $X(t)$ approaches a fixed point of the full Kostant-Toda flow as $t \rightarrow \pm \infty$,

$$
X(t) \longrightarrow \begin{cases}\epsilon+\operatorname{diag}\left(\lambda_{w(1)}, \lambda_{w(2)}, \ldots, \lambda_{w(n)}\right) & \text { as } t \rightarrow \infty \\ \epsilon+\operatorname{diag}\left(\lambda_{v(1)}, \lambda_{v(2)}, \ldots, \lambda_{v(n)}\right) & \text { as } t \rightarrow-\infty\end{cases}
$$

This can be extended to the asymptotic properties for the full Kostant-Toda hierarchy as follows: First note that for any permutation $z \in \mathcal{S}_{n}$, one can find a multi-time $\mathbf{c}=\left(c_{1}, \ldots, c_{n-1}\right) \in \mathbb{R}^{n-1}$ such that $E_{z(1)}(\mathbf{c})>E_{z(2)}(\mathbf{c})>\cdots>E_{z(n)}(\mathbf{c})$. This can be shown by considering the functions $\ell_{i}: \mathbb{R} \times \mathbb{R}^{n-1} \rightarrow \mathbb{R}$

$$
\ell_{i}\left(t_{0}, \mathbf{t}\right)=t_{0}+\lambda_{i} t_{1}+\lambda_{i}^{2} t_{2}+\cdots+\lambda_{i}^{n-1} t_{n-1}=t_{0}+\theta_{i}(\mathbf{t})=\left(t_{0}, t_{1}, \ldots, t_{n-1}\right) \cdot V_{i},
$$

where $V_{i}$ is the $i$-th column vector of the Vandermonde matrix $V$. Then one can find a point $\left(t_{0}, \mathbf{c}\right)$ such that $\ell_{z(1)}\left(t_{0}, \mathbf{c}\right)>\ell_{z(2)}\left(t_{0}, \mathbf{c}\right)>\cdots>\ell_{z(n)}\left(t_{0}, \mathbf{c}\right)$, which also implies that $E_{z(1)}(\mathbf{c})>E_{z(2)}(\mathbf{c})>$ $\cdots>E_{z(n)}(\mathbf{c})$.

Now assume that $v \leq z \leq w$. Then recall Lemma 5.6 which says $\Delta_{z \cdot[k]}^{k}(g)>0$ for all $k=$ $1, \ldots, n-1$. Note that $E_{z \cdot[k]}(\mathbf{c})$ dominates the other exponentials in the $\tau_{k}$-function (5.11) at the point $\mathbf{c}$. Then, in the direction $\mathbf{t}(s)=s \mathbf{c}$ with the limit $s \rightarrow \infty$, we have

$$
\tau_{k}(\mathbf{t}(s)) \approx d_{k} \Delta_{z \cdot[k]}^{k}(g) E_{z \cdot[k]}(\mathbf{t}(s)) \text { as } s \rightarrow \infty .
$$

Now using the formula of $f_{k}(\mathbf{t})$ in (2.22) with $t=t_{1}$, one can see that $f_{k}(\mathbf{t}(s)) \rightarrow \lambda_{z(k)}$ as $s \rightarrow \infty$, i.e. $X(\mathbf{t}(s))$ approaches the fixed point as $s \rightarrow \infty$, i.e.

$$
X(\mathbf{t}(s)) \longrightarrow \epsilon+\operatorname{diag}\left(\lambda_{z(1)}, \ldots, \lambda_{z(n)}\right) \quad \text { as } \quad s \rightarrow \infty .
$$

5.4. The moment polytope of the full Kostant-Toda lattice. We now present the image of the moment map on the full Kostant-Toda flows coming from the tnn flag variety and construct certain convex polytopes that generalize the permutohedron.

Recall that $\mathrm{L}_{i}$ denotes a weight of the standard representation of $\mathfrak{s l}_{n}$, and $\mathfrak{h}_{\mathbb{R}}^{*}$ represents the dual of the Cartan subalgebra $\mathfrak{h}_{\mathbb{R}}$, see (3.2). For $I=\left\{i_{1}, \ldots, i_{k}\right\}$, we set $\mathrm{L}(I)=\mathrm{L}_{i_{1}}+\mathrm{L}_{i_{2}}+\cdots+\mathrm{L}_{i_{k}} \in \mathfrak{h}_{\mathbb{R}}^{*}$. The moment map for the Grassmannian $\mu_{k}: G r(k, n) \rightarrow \mathfrak{h}_{\mathbb{R}}^{*}$ is defined by

$$
\mu_{k}\left(A_{k}\right):=\frac{\sum_{I \in \mathcal{M}\left(A_{k}\right)}\left|\Delta_{I}\left(A_{k}\right)\right|^{2} \mathrm{~L}(I)}{\sum_{I \in \mathcal{M}\left(A_{k}\right)}\left|\Delta_{I}\left(A_{k}\right)\right|^{2}},
$$

see e.g. 31, 30, 69.

We recall the following fundamental result of Gelfand-Goresky-MacPherson-Serganova [30] on the moment map for the Grassmannian (which in turn uses the convexity theorem of Atiyah [6] and Guillemin-Sternberg [32]).

Theorem 5.9. [30, Section 2] If $A_{k} \in G r(k, n)$ and we consider the action of the torus $\left(\mathbb{C}^{*}\right)^{n}$ on $\operatorname{Gr}(k, n)$ (which rescales columns of the matrix representing $A_{k}$ ), then the closure of the image of the moment map applied to the torus orbit of $A_{k}$ is a convex polytope

$$
\Gamma_{\mathcal{M}\left(A_{k}\right)}=\operatorname{Conv}\left\{\mathrm{L}(I) \mid \Delta_{I}\left(A_{k}\right) \neq 0 \text { i.e. } I \in \mathcal{M}\left(A_{k}\right)\right\}
$$

called a matroid polytope, whose vertices correspond to the fixed points of the action of the torus.

Remark 5.10. In representation theory, this polytope is a weight polytope of the fundamental representation of $\mathfrak{s l}_{n}$ on $\bigwedge^{k} V$, where $V$ is the standard representation. 
It should be noted that if $A_{k} \in G r(k, n)$ and we consider the action of the positive torus $\left(\mathbb{R}_{>0}\right)^{n}$ on $\operatorname{Gr}(k, n)$, the conclusion of Theorem 5.9 still holds.

The moment map for the flag variety $\mu: G / B^{+} \rightarrow \mathfrak{h}_{\mathbb{R}}^{*}$ in (3.1) can be written in the form,

$$
\mu(g):=\sum_{k=1}^{n-1} \mu_{k}\left(A_{k}\right), \quad \text { where } \quad A_{k}=\pi_{k}(g) .
$$

We now compute the image of the moment map $\mu: G / B \rightarrow \mathfrak{h}_{\mathbb{R}}^{*}$ when applied to the full KostantToda flow $\exp \left(\Theta_{C_{\Lambda}}(\mathbf{t})\right)$ on the point $n_{0} B$ of the flag variety described in (5.8).

First recall $C_{\Lambda} V=V \Lambda$ and $V g=n_{0} b_{0}$. Then we have

$$
\begin{aligned}
& \exp \left(\Theta_{C_{\Lambda}}(\mathbf{t})\right) n_{0} \cdot e_{1} \wedge \cdots \wedge e_{k}=V e^{\Theta_{\Lambda}(\mathbf{t})} g b_{0}^{-1} \cdot e_{1} \wedge \cdots \wedge e_{k} \\
= & \sum_{1 \leq i_{1}<\cdots<i_{k} \leq n} V e^{\Theta_{\Lambda}(\mathbf{t})} e_{i_{1}} \wedge \cdots \wedge e_{i_{k}}\left\langle e_{i_{1}} \wedge \cdots \wedge e_{i_{k}}, g b_{0}^{-1} \cdot e_{1} \wedge \cdots \wedge e_{k}\right\rangle \\
= & d_{k} \sum_{1 \leq i_{1}<\cdots<i_{k} \leq n} V e^{\Theta_{\Lambda}(\mathbf{t})} e_{i_{1}} \wedge \cdots \wedge e_{i_{k}}\left\langle e_{i_{1}} \wedge \cdots \wedge e_{i_{k}}, g \cdot e_{1} \wedge \cdots \wedge e_{k}\right\rangle \\
= & d_{k} \sum_{1 \leq i_{1}<\cdots<i_{k} \leq n} \Delta_{i_{1}, \ldots, i_{k}}\left(A_{k}\right) V e^{\Theta_{\Lambda}(\mathbf{t})} e_{i_{1}} \wedge \cdots \wedge e_{i_{k}} \\
= & d_{k} \sum_{1 \leq i_{1}<\cdots<i_{k} \leq n} \Delta_{i_{1}, \ldots, i_{k}}\left(A_{k} e^{\Theta_{\Lambda}(\mathbf{t})}\right) V_{i_{1}} \wedge \cdots \wedge V_{i_{k}},
\end{aligned}
$$

where $d_{k}=\left[b_{0}^{-1}\right]_{k}$ and $V_{i}=V e_{i}=\left(1, \lambda_{i}, \ldots, \lambda_{i}^{n-1}\right)^{T}$.

We now define $\varphi(\mathbf{t} ; g):=\mu\left(\exp \left(\Theta_{C_{\Lambda}}(\mathbf{t})\right) n_{0}\right)$ and $\varphi_{k}(\mathbf{t} ; g):=\mu_{k}\left(\tilde{\pi}_{k}\left(\exp \left(\Theta_{C_{\Lambda}}(\mathbf{t})\right) n_{0}\right)\right)=\mu_{k}\left(A_{k} e^{\Theta_{\Lambda}(\mathbf{t})}\right)$ with $\pi_{k}(g)=A_{k}$. Then we have

$$
\begin{gathered}
\varphi(\mathbf{t} ; g)=\sum_{k=1}^{n-1} \varphi_{k}(\mathbf{t} ; g) \quad \text { with } \quad \varphi_{k}(\mathbf{t} ; g)=\sum_{I \in \mathcal{M}\left(A_{k}\right)} \alpha_{I}^{k}(\mathbf{t} ; g) \mathrm{L}(I), \\
\quad \text { and } \quad \alpha_{I}^{k}(\mathbf{t} ; g)=\frac{\left(\Delta_{I}\left(A_{k} e^{\Theta_{\Lambda}(\mathbf{t})}\right)\right)^{2}}{\sum_{J \in \mathcal{M}\left(A_{k}\right)}\left(\Delta_{J}\left(A_{k} e^{\Theta_{\Lambda}(\mathbf{t})}\right)\right)^{2}} .
\end{gathered}
$$

Note here that $0<\alpha_{I}^{k}(\mathbf{t} ; g)<1$ and $\sum_{I \in \mathcal{M}\left(A_{k}\right)} \alpha_{I}^{k}(\mathbf{t} ; g)=1$ for each $k$.

Definition 5.11. We define the moment map image of the full Kostant-Toda flow for $g \in G_{\mathbf{v}, \mathbf{w}}^{>0}$ to be the set

$$
\mathrm{Q}_{g}=\overline{\left\{\varphi(\mathbf{t} ; g) \mid \mathbf{t} \in \mathbb{R}^{n-1}\right\}}:=\overline{\bigcup_{\mathbf{t} \in \mathbb{R}^{n-1}} \varphi(\mathbf{t} ; g)} .
$$

Here the closure is taken using the usual topology of the Euclidian norm on $\mathfrak{h}_{\mathbb{R}}^{*} \cong \mathbb{R}^{n-1}$.

Then we can show [50] that for each $k$, the image $\mathrm{Q}_{g}^{k}:=\overline{\left\{\varphi_{k}(\mathbf{t} ; g) \mid \mathbf{t} \in \mathbb{R}^{n-1}\right\}}$ is the corresponding matroid polytope from (5.15), i.e.

$$
\mathrm{Q}_{g}^{k}=\Gamma_{\mathcal{M}\left(A_{k}\right)} \quad \text { where } A_{k}=\pi_{k}(g) .
$$

Now we have the following proposition [50]:

Proposition 5.12. Let $g \in G_{\mathbf{v}_{+}, \mathbf{w}}^{>0}$. Then the moment map image $\mathrm{Q}_{g}$ of the full Kostant-Toda flow for $g$ is a Minkowski sum of matroid polytopes. More specifically, for $A_{k}=\pi_{k}(g), k=1, \ldots, n-1$, we have

$$
\mathrm{Q}_{g}=\sum_{k=1}^{n-1} \Gamma_{\mathcal{M}\left(A_{k}\right)}
$$

We also define a certain polytope which sits inside the permutohedron. 
Definition 5.13. Let $v$ and $w$ be two permutations in $\mathcal{S}_{n}$ such that $v \leq w$. We define the Bruhat interval polytope associated to $(v, w)$ to be the following convex hull:

$$
\mathrm{P}_{v, w}:=\operatorname{Conv}\left\{\mathrm{L}_{z} \in \mathfrak{h}_{\mathbb{R}}^{*} \mid v \leq z \leq w\right\} .
$$

In other words, this is the convex hull of all permutation vectors corresponding to permutations $z$ lying in the Bruhat interval $[v, w]$. In particular, if $w=w_{0}$ and $v=e$, then we have $\mathrm{P}_{e, w_{0}}=\operatorname{Perm}_{n}$. (See 76 for the further discussion on the Bruhat interval polytopes.)

Finally we have the following theorem for the moment polytope of the full Kostant-Toda flow 50 .

Theorem 5.14. Let $g \in G_{\mathbf{v}_{+}, \mathbf{w}}^{>0}$. Then the moment map image of the full Kostant-Toda flow for $g$ is the Bruhat interval polytope $\mathrm{P}_{v, w}$, i.e.

$$
\mathrm{Q}_{g}=\mathrm{P}_{v, w} .
$$

Note that from Proposition 5.12 and Theorem 5.14, we have the following remark:

Remark 5.15. The Bruhat interval polytope $\mathrm{P}_{v, w}$ is a Minkowski sum of matroid polytopes

$$
\mathrm{P}_{v, w}=\sum_{k=1}^{n-1} \Gamma_{\mathcal{M}_{k}} .
$$

Here $\mathcal{M}_{k}$ is the matroid defining the cell of $G r(k, n)_{\geq 0}$ that we obtain by projecting the cell $\mathcal{R}_{v, w}^{>0}$ of $(G / B)_{\geq 0}$ to $G r(k, n)_{\geq 0}$.

We note that each weight vector $\mathrm{L}_{i_{1}, \ldots, i_{n}}$ defined in (3.3) can be associated to the ordered set of eigenvalues,

$$
\mathrm{L}_{i_{1}, \ldots, i_{n}} \Longleftrightarrow\left(\lambda_{i_{1}}, \lambda_{i_{2}}, \ldots, \lambda_{i_{n}}\right) .
$$

This means that each vertex of the Bruhat interval polytope can be labeled by the ordered set of eigenvalues. For example, the highest weight for the permutohedron of (3.4) is given by

$$
\mathrm{L}_{1,2, \ldots, n}=\sum_{k=1}^{n}(n-k) \mathrm{L}_{k} \Longleftrightarrow\left(\lambda_{1}, \lambda_{2}, \ldots, \lambda_{n}\right) .
$$

which corresponds to the asymptotic form of $\operatorname{diag}(L)$ with $v=e$ for $t \rightarrow-\infty$. The permutohedron $\mathrm{P}_{e, w_{0}}$ with the longest element $w_{0}$ for $S L(4, \mathbb{R}) / B$ is illustrated in Fig. 5.1 (Left).

Example 5.16. Consider the $\mathfrak{s l}_{4}(\mathbb{R})$ full Kostant-Toda hierarchy. We take

$$
w=s_{2} s_{3} s_{2} s_{1} \text { and } v=s_{3},
$$

which gives

$$
w \cdot(1,2,3,4)=(4,1,3,2) \text { and } v \cdot(1,2,3,4)=(1,2,4,3) .
$$

There are eight permutations $z$ satisfying $v \leq z \leq w$, i.e.

$$
v=s_{3}, \quad s_{3} s_{2}, \quad s_{2} s_{3}, \quad s_{3} s_{1}, \quad s_{3} s_{2} s_{1}, \quad s_{2} s_{3} s_{1}, \quad s_{2} s_{3} s_{2}, \quad w=s_{2} s_{3} s_{2} s_{1} .
$$

We illustrate the moment polytopes in Figure 5.1. The vertices are labeled by the index set " $i_{1} i_{2} i_{3} i_{4}$ " of the eigenvalues $\left(\lambda_{i_{1}}, \lambda_{i_{2}}, \lambda_{i_{3}}, \lambda_{i_{4}}\right)$. The vertex with the white circle indicates the asymptotic form of $\operatorname{diag}(L)$ for $t \rightarrow-\infty$ (i.e. $(1,2,4,3)=v \cdot(1,2,3,4)$ in the right figure), and the black one indicates the asymptotic form for $t \rightarrow \infty$ (i.e. $(4,1,3,2)=w \cdot(1,2,3,4)$ in the right figure).

Remark 5.17. The moment polytopes of the full symmetric Toda hierarchy can be shown to have the same structure as these of the full Kostant-Toda hierarchy on the tnn flag variety (see [7, 17, 50]). 

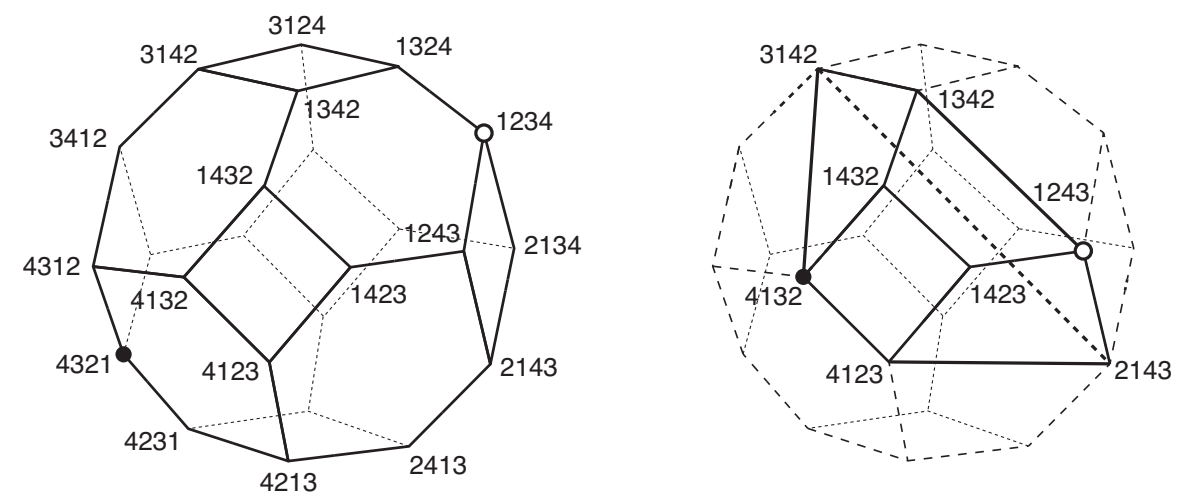

Figure 5.1. Some moment polytopes from the $\mathfrak{s l}_{4}(\mathbb{R})$ full Kostant-Toda hierachy. Each 4-digit number represents the order of the eigenvalues, e.g. $2413=$ $\left(\lambda_{2}, \lambda_{4}, \lambda_{1}, \lambda_{3}\right)$. Left: the permutohedron $\mathrm{P}_{e, w_{0}}=\mathrm{Perm}_{4}$ with $e=1234$ and $w_{0}=4321$. Right: the Bruhat interval polytope $\mathrm{P}_{v, w}$ with $w=s_{2} s_{3} s_{2} s_{1}$ and $v=s_{3}$, equivalently, they are represented as $w=4132$ and $v=1243$.

\section{The Toda lattice and integral Cohomology of Real Flag Varieties}

Here we explain how one can find the integral cohomology of real flag variety from the isospectral variety of the Toda lattice (this is an invitation to the papers [14, 15]). We consider the Toda lattice hierarchy (3.5) on the real split semi-simple Lie algebra $\mathfrak{s l}_{n}(\mathbb{R})$, and assume $X \in \mathfrak{s l}_{n}(\mathbb{R})$ to be a generic element in the tridiagonal Hessenberg form of (2.16), that is, it has all real and distinct eigenvalues (see [14, 15], for the general case associated with real split semisimple Lie algebra).

6.1. Integral cohomology of $G / B$. We begin with a brief summary of the cohomology of $G / B$ as a background for the next section where we explain how one gets the cohomology of $G / B$ from the isospectral variety of the Toda lattice associated with real split semisimple Lie group $G$.

Let us first recall the Bruhat decomposition of $G / B$,

$$
G / B=\bigsqcup_{w \in W} \Omega_{w}^{\circ} \quad \text { with } \quad \Omega_{w}^{\circ}=N w B / B .
$$

Each Bruhat (or Schubert) cell $\Omega_{w}^{\circ}$ is labeled by the element $w \in W$ and $\operatorname{codim}\left(\Omega_{w}\right)=l(w)$ where $l(w)$ represents the length of $w$. Let $\sigma_{w}$ denote the Schubert class associated to the Schubert variety $\Omega_{w}=\cup_{w \leq w^{\prime}} \Omega_{w^{\prime}}^{\circ}$. Here the Bruhat order is defined as $w \leq w^{\prime}$ iff $\Omega_{w} \supset \Omega_{w^{\prime}}$. Then we can define the chain complex,

$$
\mathcal{C}^{*}=\bigoplus_{k=0}^{l\left(w_{0}\right)} \mathcal{C}^{k} \quad \text { with } \quad \mathcal{C}^{k}=\sum_{l(w)=k} \mathbb{Z} \sigma_{w}
$$

where $w_{0}$ is the longest element of $W$, and the coboundary operators $\delta_{k}: \mathcal{C}^{k} \rightarrow \mathcal{C}^{k+1}$ is given by

$$
\delta_{k}\left(\sigma_{w}\right)=\sum_{l\left(w^{\prime}\right)=k+1}\left[w: w^{\prime}\right] \sigma_{w^{\prime}},
$$

where $\left[w: w^{\prime}\right]$ is the incidence number associated with $\sigma_{w} \stackrel{\delta_{k}}{\longrightarrow} \sigma_{w^{\prime}}$. It has been known (see [40, 16]) that the incidence number is either 0 or \pm 2 for the real flag variety $G / B$ of real split semi-simple Lie group $G$. Then the cohomology of $G / B$ can be calculated from the incidence graph $\mathcal{G}_{G / B}$ defined as follows: 
e
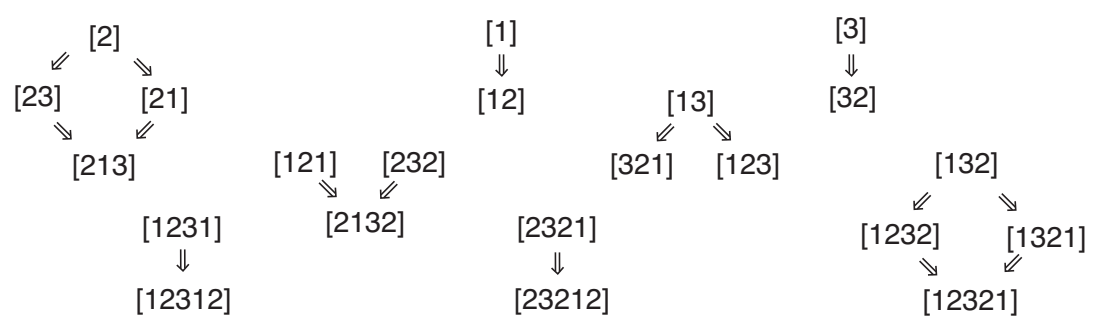

$\mathrm{w}_{0}=[123121]$

Figure 6.1 . The incidence graph $\mathcal{G}_{G / B}$ for the real flag variety $S L(4, \mathbb{R}) / B$. The Schubert class $\sigma_{w}$ is denoted by $[i j \ldots k]$ for $w=s_{i} s_{j} \ldots s_{k} . w_{0}=[123121]$ is the longest element of $W=\mathcal{S}_{4}$. The incidence numbers associated with the edges $\Rightarrow$ are \pm 2 (see also Example 8.1 in [16]).

Definition 6.1. The incidence graph $\mathcal{G}_{G / B}$ consists of the vertices labeled by $w \in W$ and the edges $\Rightarrow$ defined by

$$
w \Rightarrow w^{\prime} \quad \text { iff } \quad\left\{\begin{array}{l}
\text { (i) } w \leq w^{\prime} \\
\text { (ii) } l\left(w^{\prime}\right)=l(w)+1 \\
\text { (iii) }\left[w: w^{\prime}\right] \neq 0
\end{array}\right.
$$

The incidence number for each edge is either 0 or \pm 2 (see [16]). The integral cohomology is then calculated from the graph.

Example 6.2. In the case of $G=S L(3, \mathbb{R})$, the incidence graph and the integral cohomology are given by

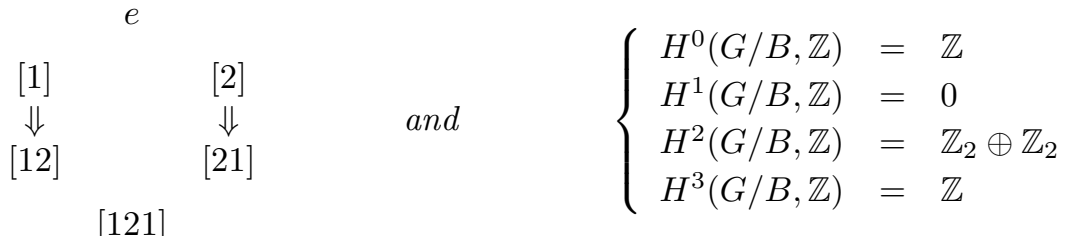

Here the Schubert classes are denoted by $[i j]$ for $w=s_{i} s_{j}$, e.g. $\delta_{1}[2]= \pm 2[21]$ for $\delta_{1} \sigma_{s_{2}}= \pm 2 \sigma_{s_{2} s_{1}}$.

In Figure 6.1, we show the incidence graph for $G / B$ with $G=S L(4, \mathbb{R})$, from which one can compute the integral cohomology [16] as

$$
\left\{\begin{array}{l}
H^{0}(G / B, \mathbb{Z})=\mathbb{Z} \\
H^{1}(G / B, \mathbb{Z})=0 \\
H^{2}(G / B, \mathbb{Z})=\mathbb{Z}_{2} \oplus \mathbb{Z}_{2} \oplus \mathbb{Z}_{2} \\
H^{3}(G / B, \mathbb{Z})=\mathbb{Z} \oplus \mathbb{Z} \oplus \mathbb{Z}_{2} \oplus \mathbb{Z}_{2} \\
H^{4}(G / B, \mathbb{Z})=\mathbb{Z}_{2} \oplus \mathbb{Z}_{2} \\
H^{5}(G / B, \mathbb{Z})=\mathbb{Z}_{2} \oplus \mathbb{Z}_{2} \oplus \mathbb{Z}_{2} \\
H^{6}(G / B, \mathbb{Z})=\mathbb{Z}
\end{array}\right.
$$

The incidence graph for the general case of real split semisimple $G$ can be found in [16]. Then the integral cohomology of $G / B$ can be computed from the incidence graph with the incidence numbers $\left[w: w^{\prime}\right]$ being 0 or \pm 2 . 
For the rational cohomology, we have

$$
H^{*}(G / B, \mathbb{Q})=H^{*}(K / T, \mathbb{Q})=H^{*}(K, \mathbb{Q})
$$

where $K$ is the maximal compact subgroup of $G$, and $T$ is the maximal torus of $G$. e.g. for $G=S L(n, \mathbb{R}), K=S O(n)$, and $T=\operatorname{diag}( \pm 1, \ldots, \pm 1)$ (see Proposition 6.3 in 15). It is well known that the cohomology ring $H^{*}(K, \mathbb{Q})$ of compact connected group $K$ of rank $l$ is given by the exterior product algebra,

$$
H^{*}(K, \mathbb{Q})=\bigwedge_{\mathbb{Q}}\left\{x_{m_{1}}, x_{m_{2}}, \ldots, x_{m_{l}}\right\},
$$

where $\left\{x_{m_{1}}, \ldots, x_{m_{l}}\right\}$ are the generators of the exterior product representation with $\operatorname{deg}\left(x_{m_{i}}\right)=m_{i}$ (odd) for $i=1, \ldots, l$ and $m_{1}+\cdots+m_{l}=\operatorname{dim}(K)$ (see for example [9]). In the case of $K=S O(n)$, we have,

(a) for $n=2 m+1$,

$$
H^{*}(S O(2 m+1), \mathbb{Q})=\bigwedge_{\mathbb{Q}}\left(x_{3}, x_{7}, \ldots, x_{4 m-1}\right)
$$

(b) for $n=2 m$,

$$
H^{*}(S O(2 m), \mathbb{Q})=\bigwedge_{\mathbb{Q}}\left(x_{3}, x_{7}, \ldots, x_{4 m-5}, y_{2 m-1}\right) .
$$

Note here that the generators include the additional $y_{2 m-1}$. For example, $H^{*}(S O(4), \mathbb{Q})$ is generated by two elements $x_{3}, y_{3}$ of the same degree, $V=\mathbb{Q} x_{3}+\mathbb{Q} y_{3}$ and $\wedge^{2} V=\mathbb{Q} x_{3} \wedge y_{3}$.

We also note that the number of points on the finite Chevalley group $K\left(\mathbb{F}_{q}\right)$ of the compact connected group $K$ is given by certain polynomial of $q$. Here $\mathbb{F}_{q}$ is a finite field with $q$ elements. Although this polynomial can be computed by using the Lefschetz fixed point theorem for the Frobenius map $\Phi: K\left(\overline{\mathbb{F}}_{q}\right) \rightarrow K\left(\overline{\mathbb{F}}_{q}\right), x \mapsto x^{q}$, we here give an elementary calculation to find those polynomials for $K=S O(n)$ (see also [14]). As we show in the next section, those polynomials are also related to the indefinite Toda lattice of Section 2.4 [45, 46].

Let us first assume that $q$ is a power of a prime number $p \neq 2$, such that in $\mathbb{F}_{q}$ the polynomial $x^{2}+1$ is not irreducible, i.e. $\sqrt{-1} \in \mathbb{F}_{q}$. Then we have the following results for $\left|S^{n}\left(\mathbb{F}_{q}\right)\right|$, the number of $\mathbb{F}_{q}$ points on $S^{n}$ :

$$
\left|S^{n}\left(\mathbb{F}_{q}\right)\right|=\left\{\begin{array}{lll}
q^{m-1}\left(q^{m}-1\right) & \text { if } & n=2 m-1, \\
q^{m}\left(q^{m}+1\right) & \text { if } & n=2 m .
\end{array}\right.
$$

This can be shown as follows: Let us first consider the case $n=1$, i.e.

$$
S^{1}\left(\mathbb{F}_{q}\right)=\left\{(x, y) \in \mathbb{F}_{q}^{2}: x^{2}+y^{2}=1\right\} .
$$

Then using the formulae for the stereographic projection; $x=\frac{2 u}{u^{2}+1}, y=\frac{u^{2}-1}{u^{2}+1}$ with $y \neq 1$ and $\left\{u \in \mathbb{F}_{q}: u^{2}+1 \neq 0\right\}$. Since $\sqrt{-1} \in \mathbb{F}_{q}$, we have 2 points in $\left\{u^{2}+1=0\right\}$. Counting the point $(0,1)$, the north pole, we have $\left|S^{1}\left(\mathbb{F}_{q}\right)\right|=q-2+1=q-1$. Now consider the case $n=2$, we have $x=\frac{2 u_{1}}{u_{1}^{2}+u_{2}^{2}+1}, y=\frac{2 u_{2}}{u_{1}^{2}+u_{2}^{2}+1}, z=\frac{u_{1}^{2}+u_{2}^{2}-1}{u_{1}^{2}+u_{2}^{2}+1}$ with $z \neq 1$ and $\left\{\left(u_{1}, u_{2}\right) \in \mathbb{F}_{q}^{2}: u_{1}^{2}+u_{2}^{2}+1 \neq 0\right\}$. This gives $q^{2}-(q-1)$ points (note $(q-1)$ is the number of points in $\left.u_{1}^{2}+u_{2}^{2}+1=0\right)$. We now add the points of the north pole $(x, y, 1)$ with $x^{2}+y^{2}=0$. This gives $2(q-1)+1$, where $2(q-1)$ for $x= \pm \sqrt{-1} y \neq 0$ and 1 for $(0,0,1)$. Then we have $\left|S^{2}\left(\mathbb{F}_{q}\right)\right|=q^{2}-(q-1)+2(q-1)+1=q(q+1)$. Using the induction, one can show that the number of points in the north pole is given by $\mid\left\{\left(x_{1}, \ldots, x_{2 m-1}\right) \in \mathbb{F}_{q}^{2 m-1}\right.$ : $\left.x_{1}^{2}+\cdots+x_{2 m-1}^{2}=0\right\} \mid=q^{2 m-2}$ and $\left.\mid\left\{x_{1}, \ldots, x_{2 m}\right) \in \mathbb{F}_{q}^{2 m}: x_{1}^{2}+\cdots+x_{2 m}^{2}=0\right\} \mid=q^{2 m-1}+q^{m}-q^{m-1}$. Then one can obtain the above formulae for $\left|S^{n}\left(\mathbb{F}_{q}\right)\right|$. 
We can now find the number of $\mathbb{F}_{q}$ points of finite Chevalley group $S O\left(n, \mathbb{F}_{q}\right)$ : First recall that $S O(n+1) / S O(n) \cong S^{n}$. Then we obtain

$$
\left|S O\left(n, \mathbb{F}_{q}\right)\right|=\prod_{k=1}^{n}\left|S^{n-k}\left(\mathbb{F}_{q}\right)\right|,
$$

which leads to the results [9]:

(a) For $n=2 m$,

$$
\left|S O\left(2 m, \mathbb{F}_{q}\right)\right|=2 q^{m(m-1)}\left(q^{2}-1\right)\left(q^{4}-1\right) \cdots\left(q^{2 m-2}-1\right)\left(q^{m}-1\right) .
$$

(b) For $n=2 m+1$,

$$
\left|S O\left(2 m+1, \mathbb{F}_{q}\right)\right|=2 q^{m^{2}}\left(q^{2}-1\right)\left(q^{4}-1\right) \cdots\left(q^{2 m}-1\right) .
$$

In general, the number of $\mathbb{F}_{q}$ points on the compact group $K$ can be expressed by (see e.g. 9])

$$
\left|K\left(\mathbb{F}_{q}\right)\right|=q^{r} p(q) \quad \text { with } \quad p(q)=\prod_{i=1}^{l}\left(q^{d_{i}}-1\right),
$$

where $d_{i}$ 's are degree of basic Weyl group invariant polynomials for $K$ given by $d_{i}=\left(m_{i}+1\right) / 2$, and $r=\operatorname{dim}(K)-\operatorname{deg}(p(q))$. In the next section, we show that those polynomials can be reproduced by counting the blow-ups in the solution of the indefinite Toda lattice (see 14 for the general case).

6.2. Blow-ups of the indefinite Toda lattice on $G$ and the cohomology of $G / B$. Now we show how to obtain the cohomology of $G / B$ from the moment polytope of the indefinite Toda lattice of Section 2.4

First note that the $\tau$-functions can change their signs if some (but not all) of $\sigma_{i}$ 's are negative. This can be seen from (2.18), and implies that the solution blows up for some time $t_{1}=\bar{t}_{1}$, (see also (2.22)). The explicit form of the $\tau$-functions can be obtained from (2.21), and they are expressed by (see also Proposition 3.1 in [46]),

$$
\tau_{k}(t)=\sum_{1 \leq j_{1}<\cdots<j_{k} \leq n} \sigma_{j_{1}} \cdots \sigma_{j_{k}} K\left(j_{1}, \ldots, j_{k}\right) \exp \left(\sum_{i=1}^{k} \lambda_{j_{i}} t\right),
$$

where $K\left(j_{1}, \ldots, j_{k}\right)$ are positive and given by

$$
K\left(j_{1}, \ldots, j_{k}\right)=\left(\varphi^{0}\left(\lambda_{j_{1}}\right) \cdots \varphi^{0}\left(\lambda_{j_{k}}\right)\right)^{2}\left|\begin{array}{ccc}
1 & \cdots & 1 \\
\vdots & \ddots & \vdots \\
\lambda_{j_{1}}^{k-1} & \cdots & \lambda_{j_{k}}^{k-1}
\end{array}\right|^{2}>0 .
$$

As the simplest case, let us consider the $\mathfrak{s l}_{2}(\mathbb{R})$ indefinite Toda lattice: We have one $\tau$-function,

$$
\tau_{1}(t)=\sigma_{1} \rho_{1} e^{\lambda_{1} t}+\sigma_{2} \rho_{2} e^{\lambda_{2} t} .
$$

If $\sigma_{1} \sigma_{2}=-1, \tau_{1}(t)$ has zero at a time $t=\frac{1}{\lambda_{2}-\lambda_{1}} \ln \left(\frac{\rho_{1}}{\rho_{2}}\right)$, that is, we have a blow-up in the solution. The image of the moment map $\mu\left(\tau_{1}\right)$ is given by a line segment whose end points correspond to the weights $\mathrm{L}_{1}$ and $\mathrm{L}_{2}=-\mathrm{L}_{1}$. Although the dynamics are so different in the cases $\sigma_{1} \sigma_{2}>0$ and $\sigma_{1} \sigma_{2}<0$, the moment polytope (a line segment) is independent of the signs of the $\sigma_{i}$ 's. Notice that $\sigma_{1} \sigma_{2}=\operatorname{sgn}\left(g_{1}\right)$, and in general, if $\operatorname{sgn}\left(g_{k}\right)<0$ for some $k$, then the solution blows up sometime in $\mathbb{R}$.

In order to find the general pattern of the sign changes in $\left(g_{1}(t), \ldots, g_{n-1}(t)\right)$ of the matrix $X$ in (2.16) with (2.18), we first recall that the isospectral variety is characterized by the moment polytope $\mathcal{M}_{\epsilon}$ whose vertices are given by the orbit of Weyl group action. Here the set of signs $\epsilon=\left(\epsilon_{1}, \ldots, \epsilon_{n-1}\right)$ is defined by the signs of $g_{i}$ for $t \rightarrow-\infty$. From the ordering $\lambda_{1}<\cdots<\lambda_{n}$, we 
first see that $\tau_{k}(t) \approx \sigma_{1} \cdots \sigma_{k} K(1, \ldots, k) \exp \left(\left(\lambda_{1}+\cdots+\lambda_{k}\right) t\right)$. Then from the definition of $g_{k}(t)$ in (2.22), i.e. $g_{k}=\tau_{k-1} \tau_{k+1} / \tau_{k}^{2}$, the sign of $g_{k}(t)$ for $t \rightarrow-\infty$ is given by

$$
\epsilon_{k}=\operatorname{sgn}\left(g_{k}\right)=\sigma_{k} \sigma_{k+1} \quad \text { for } \quad k=1, \ldots, n-1 \text {. }
$$

Then from the moment map (3.1), one notes that the moment polytope given as the image of the moment map $\mu\left(\mathcal{M}_{\epsilon}\right)$ is independent of the sign set $\epsilon$. However the dynamics of the Toda lattice with a different $\epsilon$ is quite different, and the solution with at least one $\epsilon_{k}<0$ has a blow-up at some $t \in \mathbb{R}$.

We now consider each edge of the polytope which corresponds to an $\mathfrak{s l}_{2}(\mathbb{R})$ indefinite Toda lattice, that is, where $g_{j} \neq 0$ for only one $j$. This edge can be also expressed by a simple reflection $s_{j} \in W$. Since the simple reflection $s_{j}$ exchanges $\sigma_{j}$ and $\sigma_{j+1}$, we have an action of $s_{j}$ on all the signs $\epsilon_{k}$, $s_{j}: \epsilon_{k} \rightarrow \epsilon_{k}^{\prime}$

$$
\epsilon_{k}^{\prime}=s_{j}\left(\epsilon_{k}\right)= \begin{cases}\epsilon_{k} \epsilon_{k-1} & \text { if } \quad j=k-1 \\ \epsilon_{k} \epsilon_{k+1} & \text { if } j=k+1 \\ \epsilon_{k} & \text { if } j=k \text {, or }|j-k|>1\end{cases}
$$

which can be also shown directly from the form of $\tau_{k}(t)$ in $(\underline{6.3})$. This formula can be extended to the indefinite Toda lattice on any real split semisimple Lie algebras, and we have (see (4.4) and Proposition 3.16 in 10 ):

Proposition 6.3. Let $\epsilon_{j}=\operatorname{sgn}\left(g_{j}\right)$ for $j=1, \ldots, n-1$. Then the Weyl group action on the signs is given by

$$
s_{j}: \epsilon_{k} \longmapsto \epsilon_{k} \epsilon_{j}^{-C_{k j}},
$$

where $\left(C_{i j}\right)_{1 \leq i, j \leq n-1}$ is the Cartan matrix of $\mathfrak{s l}_{n}(\mathbb{R})$.

With this $W$-action on the signs $\epsilon=\left(\epsilon_{1}, \ldots, \epsilon_{n-1}\right)$ with $\epsilon_{k}=\operatorname{sgn}\left(g_{k}\right)$ at each vertex of the polytope, we now define the relation between the vertices labeled by $w$ and $w^{\prime}=w s_{i}$ as follows: Notice that if $\epsilon_{i}=+$, then $\left(\epsilon_{1}, \cdots, \epsilon_{n-1}\right)$ remains the same under $s_{i}$-action. Then we write

$$
w \Longrightarrow w^{\prime} \quad \text { with } \quad w^{\prime}=w s_{i} \text {. }
$$

The following definition gives the number of blow-ups in the Toda orbit from the top vertex $e$ to the vertex labeled by $w \in W$ : Choose a reduced expression $w=s_{j_{1}} \cdots s_{j_{k}}$, and consider the sequence of the signs at the orbit given by $w$-action,

$$
\epsilon \longrightarrow s_{j_{1}} \epsilon \longrightarrow s_{j_{2}} s_{j_{1}} \epsilon \longrightarrow \cdots \longrightarrow w^{-1} \epsilon .
$$

We then define the function $\eta(w, \epsilon)$ as the number of $\rightarrow$ which are not of the form $\Rightarrow$. The number $\eta\left(w_{0}, \epsilon\right)$ for the longest element $w_{0}$ gives the total number of blow-ups along the Toda flow in the polytope of $\mathcal{M}_{\epsilon}$. Whenever $\epsilon=(-, \ldots,-)$, we just denote $\eta(w, \epsilon)=\eta(w)$. This number $\eta(w, \epsilon)$ does not depend on the choice of the reduced expression of $w$ (see Corollary 5.2 in [14]). Hence the number of blow-up points along the trajectories in the edges of the polytope is independent of the trajectory parametrized by the reduced expression. In Figure 2.2 we illustrate the numbers $\eta(w, \epsilon)$ for the $\mathfrak{s l}_{3}(\mathbb{R})$ indefinite Toda lattice. For example, on $\mathcal{M}_{--}$, we have $\eta(e)=0, \eta\left(s_{1}\right)=$ $\eta\left(s_{2}\right)=\eta\left(s_{1} s_{2}\right)=\eta\left(s_{2} s_{1}\right)=1$ and $\eta\left(s_{1} s_{2} s_{1}\right)=2$, i.e the total number of blow-ups is 2 . We also illustrate this for the $\mathfrak{s l}_{4}(\mathbb{R})$ Toda lattice in Figure 6.2. Along the path shown in this Figure, we have $\eta(e)=0, \eta([2])=\eta([21])=\eta([213])=1, \eta([2132])=2, \eta([21323])=3$ and $\eta\left(w_{0}\right)=4$, where $[i j \cdots k]=s_{i} s_{j} \cdots s_{k}$, and note $[21323]=[12312]$.

In general, the total number of blow-ups $\eta\left(w_{0}, \epsilon\right)$ depends only the initial signs $\epsilon=\left(\epsilon_{1}, \ldots, \epsilon_{n-1}\right)$ with $\epsilon_{i}=\operatorname{sgn}\left(g_{i}(t)\right)$ for $t \rightarrow-\infty$, which is given by $\epsilon_{i}=\sigma_{i} \sigma_{i+1}$. Then in the case of $\mathfrak{s l}_{n}(\mathbb{R})$ indefinite Toda lattice, the number $\eta\left(w_{0}, \epsilon\right)=m(n-m)$ where $m$ is the total number of negative $\sigma_{i}$ 's (Proposition 3.3 in [46]). In particular, the maximum number of blow-ups occurs in the case 

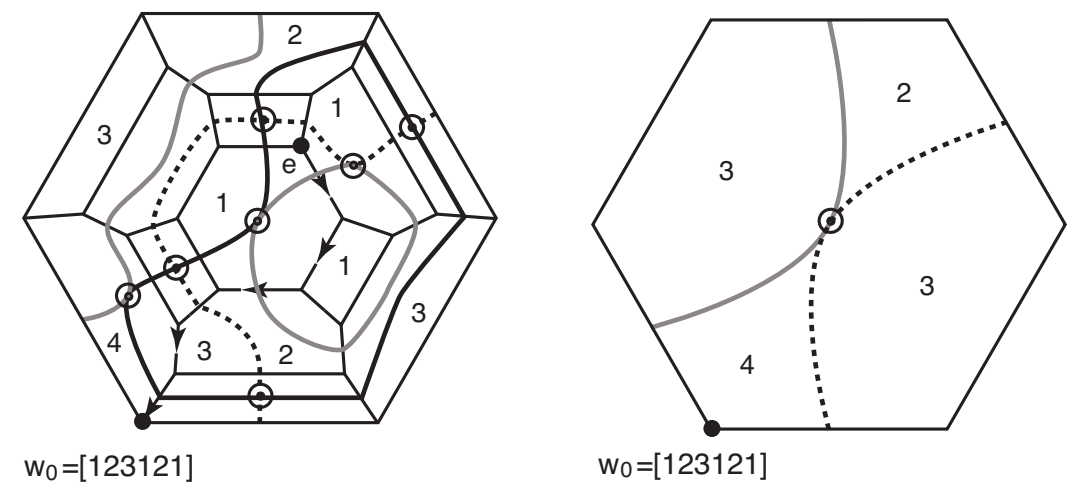

Figure 6.2. The moment polytope $\mathcal{M}_{---}$for the $\mathfrak{s l}_{4}(\mathbb{R})$ indefinite Toda lattice (the right figure is the back view of the left one). The divisors defined by the set of zero points for the $\tau$-functions are shown by the dotted curve for $\left\{\tau_{1}=0\right\}$, by the light curve for $\left\{\tau_{2}=0\right\}$, and by the dark one for $\left\{\tau_{3}=0\right\}$. The double circles indicate the divisors with $\left\{\tau_{i}=0\right\} \cap\left\{\tau_{j}=0\right\}$, which are all connected at the center of the polytope corresponding to the point with $\left\{\tau_{1}=\tau_{2}=\tau_{3}=0\right\}$. The numbers in the polytope indicate the number of blow-ups along the flow. An example of a path from the top vertex $e$ to the bottom vertex $w_{0}$, the longest element of $\mathcal{S}_{4}$, is shown by directed edges.

where $\epsilon=(-, \ldots,-)$, and it is given by $[(n+1) / 2](n-[(n+1) / 2])$. Those numbers $\eta\left(w_{0}, \epsilon\right)$ are related to the polynomials given in (6.2) appearing in $\mathbb{F}_{q}$ points on certain compact groups $K$.

We now introduce polynomials in terms of the numbers $\eta(w, \epsilon)$, which play a key role for counting the number of blow-ups and give a surprising connection to the rational cohomology of the maximal compact subgroup $S O(n)$ (Definition 3.1 in [14]).

Definition 6.4. We define a monic polynomial associated to the polytope $\mathcal{M}_{\epsilon}$,

$$
p(q, \epsilon)=(-1)^{l\left(w_{0}\right)} \sum_{w \in W}(-1)^{l(w)} q^{\eta(w, \epsilon)},
$$

where $l(w)$ indicates the length of $w$. Notice that the degree of $p(q, \epsilon)$, denoted by $\operatorname{deg}(p(q, \epsilon))$, is the total number of blow-ups, i.e. $\eta\left(w_{0}, \epsilon\right)=\operatorname{deg}(p(q, \epsilon))$. For the case $\epsilon=(-, \ldots,-)$, we simply denote it by $p(q)$.

Example 6.5. In the case of the $\mathfrak{s l}_{2}(\mathbb{R})$ Toda lattice,

(a) for $\epsilon=(+)$, we have $e \Rightarrow s_{1}$ which gives $p(q,+)=0$,

(b) for $\epsilon=(-)$, we have a blow-up between e and $s_{1}$, hence $p(q,-)=q-1$.

Recall from the previous section that the polynomial $p(q)=p(q,-)$ appears in $\left|S O\left(2, \mathbb{F}_{q}\right)\right|=q-1$.

In the case of the $\mathfrak{s l}_{3}(\mathbb{R})$ Toda lattice, from Figure 2.2,

(a) for all the cases of $\epsilon=\left(\epsilon_{1}, \epsilon_{2}\right)$ except $(-,-)$, we have $p(q, \epsilon)=0$.

(b) for $\epsilon=(-,-)$, we have $p(q)=q^{2}-1$.

Note again that the polynomial $p(q)$ appears in $\left|S O\left(3, \mathbb{F}_{q}\right)\right|=q\left(q^{2}-1\right)$.

In the case of $\mathfrak{s l}_{4}(\mathbb{R})$, we have, from Figure 6.2,

(a) for all $\epsilon=\left(\epsilon_{1}, \epsilon_{2}, \epsilon_{3}\right)$ except $(-,-,-), p(q, \epsilon)=0$.

(b) for $\epsilon=(-,-,-), p(q)=q^{4}-2 q^{2}+1=\left(q^{2}-1\right)^{2}$.

Again note that $\left|S O\left(4, \mathbb{F}_{q}\right)\right|=q^{2}\left(q^{2}-1\right)^{2}$. 
Casian and Kodama prove that the polynomial $p(q)$ for $\mathcal{M}_{\epsilon}$ with $\epsilon=(-, \ldots,-)$ in Definition 6.4 agrees with the polynomial $p(q)$ in $\left|K\left(\mathbb{F}_{q}\right)\right|$ in (6.2) where $K$ is the maximal compact subgroup of real split semisimple Lie group $G$ for the Toda lattice (Theorem 6.5 in [14]).

Thus the polynomial $p(q)$ contains all the information on the $\mathbb{F}_{q}$ points on the compact subgroup $K$ of $G$, which is also related to the rational cohomology, i.e. $H^{*}(K, \mathbb{Q})=H^{*}(G / B, \mathbb{Q})$ (see (6.1) ). Now recall that the integral cohomology of the real flag variety $G / B$ is obtained by the incidence graph $\mathcal{G}_{G / B}$ in Definition 6.1] In [14, Casian and Kodama show that the graph $\mathcal{G}_{G / B}$ can be obtained from the blow-ups of the Toda flow. They define a graph $\mathcal{G}_{\epsilon}$ associated to the blow-ups as follows:

Definition 6.6. The graph $\mathcal{G}_{\epsilon}$ consists of vertices labeled by the elements of the Weyl group $W$ and oriented edges $\Rightarrow$. The edges are defined as follows:

$$
w_{1} \Rightarrow w_{2} \quad \text { iff } \quad\left\{\begin{array}{l}
\text { (a) } w_{1} \leq w_{2} \text { (Bruhat order) } \\
\text { (b) } l\left(w_{1}\right)=l\left(w_{2}\right)+1 \\
\text { (c) } \eta\left(w_{1}, \epsilon\right)=\eta\left(w_{2}, \epsilon\right) \\
\text { (d) } w_{1}^{-1} \epsilon=w_{2}^{-1} \epsilon
\end{array}\right.
$$

When $\epsilon=(-, \ldots,-)$, we simply denote $\mathcal{G}=\mathcal{G}_{\epsilon}$.

Then they prove that $\mathcal{G}_{\epsilon}$ with $\epsilon=(-, \ldots,-)$ is equivalent to $\mathcal{G}_{G / B}$ (Theorem 3.5 in 14 which is the main theorem in the paper). For example, the graph $\mathcal{G}$ associated with Figure 6.2 agrees with the incidence graph $\mathcal{G}_{G / B}$ given in Figure 6.1. The proof of the equivalence $\mathcal{G}_{G / B}=\mathcal{G}$ contains several technical steps, which are beyond the scope of this review.

\section{REFERENCES}

[1] M. Adler and P. van Moerbeke, Toda versus Pfaff lattice and related polynomials, Duke Math. Journal, 112:1-58 (2002)

[2] M. Adler and P. van Moerbeke, Completely integrable systems, Euclidian Lie algebras and curves, Adv. Math. 38: 267-317 (1980)

[3] M. Adler and P. van Moerbeke, Linearization of Hamiltonian systems, Jacobi varieties and representation theory, Adv. Math. 38: 318-379 (1980)

[4] M. Adler and P. van Moerbeke, The Toda lattices, Dynkin diagrams, singularities and Abelian varieties, Invent. Math. 103: 223-278 (1991)

[5] V. I. Arnold, Mathematical Methods of Classical Mechanics, 2nd ed., Graduate Texts in Mathematics 60, Springer-Verlag, 1989

[6] M. B. Atiyah, Convexity and Commuting Hamiltonians, Bull. London Math. Soc. 14:1-15 (1982)

[7] A. M. Bloch and M. Gekhtman, Hamiltonian and gradient structures in the Toda flows, J. Geom. Phys. 27: 230-248 (1998)

[8] O. I. Bogoyavlensky, On perturbations of the periodic Toda lattice, Comm. Math. Phys. 51: 201-209 (1976)

[9] R. Carter, Simple Groups of Lie type, (Wiley Classical Library Edition, London, New York, Sidney, Toronto, 1989)

[10] L. Casian and Y. Kodama, Toda lattice and toric varieties for real split semisimple Lie algebras, Pacific J. Math. 207: $77-123(2002)$

[11] L. Casian and Y. Kodama, Blow-ups of the Toda lattices and their intersections with the Bruhat cells, Comtemporary Math. 301: 283-310 (2002)

[12] L. Casian and Y. Kodama, Twisted Tomei manifolds and the Toda lattices, Contemp. Math. 309: 1-19 (2002)

[13] L. Casian and Y. Kodama, Compactification of the isospectral varieties of nilpotent Toda lattices, RIMS Kokyuroku, Kyoto University, 1400: 39-87 (2004)

[14] L. Casian and Y. Kodama, Toda lattice, cohomology of compact Lie groups and finite Chevalley groups, Invent. Math. 165: 163-208 (2006)

[15] L. Casian and Y. Kodama, Singular structure of Toda lattices and cohomology of certain compact Lie groups, J. Comp. Appl. Math. 202: 56-79 (2007)

[16] L. Casian and R. Stanton, Schubert cells and representation theory, Invent. Math. 137: 461-539 (1999)

[17] Y. B. Chernyakov, G. I. Sharygin and A. S. Sorin, Bruhat order in full symmetric Toda system, Commun. Math. Phys. 330: 367-399 (2014). 
[18] P. Deift, L. C. Li, T. Nanda, and C. Tomei, The Toda flow on a generic orbit is integrable, CPAM 39: 183-232 (1986)

[19] V. Deodhar, On some geometric aspects of Bruhat orderings. I. A finer decomposition of Bruhat cells, Invent. Math. 79(3): 499-511 (1985).

[20] B. A. Dubrovin, Theta functions and nonlinear equations, Russ. Math. Sur. 36, No.2, 11-92 (1981)

[21] N. M. Ercolani and H. Flaschka and L. Haine, Painleve Balances and Dressing Transformations, In: Painleve Transcendents, NATO ASI series, Series B, Physics 278 (1991)

[22] N. Ercolani, H. Flaschka, and S. Singer, The geometry of the full Kostant-Toda lattice In: Integrable Systems, Vol. 115 of Progress in Mathematics 181-226, Birkhäuser (1993)

[23] L. Fehér and I. Tsutsui, Regularization of Toda lattices by Hamiltonian reduction, J. Geom. Phys. 21(2): 97-135 (1997)

[24] H. Flaschka, The Toda lattice. II. Existence of integrals, Phys. Rev. B 9(4): 1924-1925 (1974)

[25] H. Flaschka, On the Toda lattice. II, Prog. Theor. Phys. 51(3): 703-716 (1974)

[26] H. Flaschka, Integrable systems and torus actions, in Lectures on Integrable Systems: In Memory of Jean-Louis Verdier, Eds. O Babelon, P. Cartier and Y. Kosmann-Schwarzbach, 43-101 (World Scientific, 1994)

[27] H. Flaschka and L. Haine, Torus orbits in G/P, Pacific J. Math. 149(2): 251-292 (1991)

[28] H. Flaschka and L. Haine, Varietes de drapeaux et reseaux de Toda, Math. Z. 208: 545-556 (1991)

[29] M. Gekhtman and M. Shapiro, Noncommutative and commutative integrability of generic Toda flows in simple Lie algebras, Comm. Pure \& Appl. Math. 52: 53-84 (1999)

[30] I. M. Gelfand, R. M. Goresky, R. D. MacPherson and V. V Serganova, Combinatorial geometries, convex polyhedra, and Schubert cells, Adv. Math. 63: 301-316 (1987)

[31] I. M. Gelfand and V. V. Serganova, Combinatorial geometries and torus strata on homogeneous compact manifolds, Usp. Mat. Nauk. 42(2):107-134 (1987)

[32] I. M. Guillemin and S. Sternberg, Convexity properties of the moment mapping, Invent. Math. 67: 491-513 (1982)

[33] F. Gesztesy, H. Holden, B. Simon and Z. Zhao, On the Toda lattice and Kac-van Moerbeke systems, Trans. AMS, 339: 849-868 (1993)

[34] M. A. Guest, Harmonic Maps, Loop Groups, and Integrable Systems, London Mathematical Society Student Texts 38 (Cambridge University Press,1997)

[35] M. Henon, Integrals of the Toda lattice, Phys. Rev. B 9:1921-1923 (1974)

[36] R. Hirota, The Direct Method in Soliton Theory, (Cambridge University Press, Cambridge, 2004)

[37] M. Kac and P. van Moerbeke, On an explicitly soluble system of nonlinear differential equations related to certain Toda lattices, Adv. Math. 16: 160-169 (1975)

[38] B. B. Kadomtsev and V. I. Petviashvili, On the stability of solitary waves in weakly dispersing media, Sov. Phys. Doklady, 15: 539-541 (1970)

[39] F. C. Kirwan, Cohomology of quotients in symplectic and algebraic geometry Math. Notes 31, (Princeton University Press, 1984)

[40] R. R. Kocherlakota, Integral homology of real flag manifolds and loop spaces of symmetric spaces, Adv. Math. 110: 1-46 (1995)

[41] Y. Kodama, KP solitons and the Grassmannians, Springer Brief in Mathematical Physics, vol. 22, (Springer, 2017).

[42] Y. Kodama and K. T-R McLaughlin, Explicit integration of the full symmetric Toda hierarchy and the sorting property, Lett. Math. Phys. 37:37-47 (1996)

[43] Y. Kodama and V. U. Pierce, Geometry of the Pfaff lattices, Inter. Math. Res. Notes, 120, 55 pages, (2007)

[44] Y. Kodama and V. U. Pierce, The Pfaff lattice on symplectic matrices, J. Phys. A: Math. Theor. $43: 055206$ (22pp) (2010).

[45] Y. Kodama and J. Ye, Toda hierarchy with indefinite metric, Physica D 91:321-339 (1996)

[46] Y. Kodama and J. Ye, Toda lattices with indefinite metric II: topology of the iso-spectral manifolds, Physica D 121:89-108 (1998)

[47] Y. Kodama and J. Ye, Iso-spectral deformations of general matrix and their reductions on Lie algebras, Commun. Math. Phys. 178:765-788 (1996)

[48] Y. Kodama, L. Williams, KP solitons and total positivity for the Grassmannian, Invent. Math. 198, (2014) 637-699.

[49] Y. Kodama, L. Williams, The Deodhar decomposition of the Grassmannian and the regularity of KP solitons, Adv. Math. 244, (2013) 979-1032.

[50] Y.Kodama, L.Williams, The full Kostant-Toda hierarchy on the positive flag variety, Commun. Math. Phys. 335 : 247-283 (2015).

[51] B. M. Kostant, On Whittaker vectors and representation theory, Invent. Math. 48:101-184 (1978) 
[52] B. M. Kostant, The solution to a generalized Toda lattice and representation theory, Adv. Math. 34:195-338 (1979)

[53] P. D. Lax, Integrals of nonlinear equations of evolution and solitary waves, Comm. Pure Appl. Math. 21:467-490 (1968)

[54] G. Lusztig, Total positivity in partial flag manifolds, Representation Theory, 2 (1998) 70-78.

[55] G. Lusztig, Total positivity in reductive groups, in: Lie theory and geometry: in honor of Bertram Kostant, Progress in Mathematics 123, Birkhauser, 1994.

[56] S. V. Manakov, Complete integrability and stochastization of discrete dynamical systems, Sov. Phys. ZETP 40 : 269-274 (1975)

[57] R. Marsh and K. Rietsch, Parametrizations of flag varieties, Representation Theory, 8: 212-242 (2004).

[58] T. Miwa, M. Jimbo and E. Date, Solitons: Differential equations, symmetries and infinite dimensional algebras, Cambridge Tracts in Mathematics 135 (Cambridge University Press, 2000)

[59] J. Moser, Finitely many mass points on the line under the influence of an exponential potential - an integrable system, In: Dynamical Systems, Theory and Applications, Lecture Notes in Physics, Vol. 38, Springer, 1975, p. 467-497, 1975

[60] A. M. Perelomov, Integrable systems of classical mechanics and Lie algebras, (Birkhäuser, Basel-Boston-Berlin, 1990)

[61] A. G. Reyman, Integrable Hamiltonian systems connected with graded Lie algebras, Zap. Nauch. Sem. LOMI 95:3-54 (1980)

[62] A. G. Reyman and M. A. Semenov-Tian-Shantsky, Reduction of Hamiltonian systems, affine Lie algebras and Lax equations, Invent. Math. 54:81-100 (1979)

[63] A. G. Reyman and M. A. Semenov-Tian-Shansky, Group-theoretical methods in the theory of finite-dimensional integrable systems, Encyclopaedia of Mathematical Sciences, Vol. 16, "Dynamical Systems VII" (Springer-Verlag, Berlin Heidelberg, 1994)

[64] K. Rietsch, Total positivity and real flag varieties, Ph.D. Dissertation, MIT, 1998.

[65] B. A. Shipman, On the geometry of certain isospectral sets in the full Kostant-Toda lattice, Pac. J. Math. 181(1):159-185 (1997)

[66] B. A. Shipman, Monodromy near the singular level set in the $S L(2$, C) Toda lattice, Phys. Lett. A 239:246-250 (1998)

[67] B. A. Shipman, A symmetry of order two in the full Kostant-Toda lattice, J. Alg. 215:682-693 (1999)

[68] B. A. Shipman, The geometry of the full Kostant-Toda lattice of $s l(4, \mathbf{C})$, Journal of Geometry and Physics 33:295-325 (2000)

[69] B. A. Shipman, Nongeneric flows in the full Toda lattice, In: Contemporary Mathematics: Integrable Systems, Topology, and Physics 309:219-249, American Mathematical Society (2002)

[70] B. A. Shipman, Compactified isospectral sets of complex tridiagonal Hessenberg matrices, In: Dynamical Systems and Differential Equations, Eds. W. Feng, S. Hu, and X. Lu., American Institute of Mathematical Sciences, 788797 (2003)

[71] W. W. Symes, Hamiltonian group actions and integrable systems, Physica 1D:275-280 (1980)

[72] W. W. Symes, The QR algorithm and scattering for the finite nonperiodic Toda lattice, Physica D:275-280 (1982)

[73] M. Toda, Vibration of a chain with nonlinear interaction, J. Phys. Soc. Japan 22(2):431-436 (1967)

[74] M. Toda, Wave propagation in anharmonic lattices, J. Phys. Soc. Japan 23(3):501-506 (1967)

[75] C. Tomei, The topology of isospectral manifolds of tridiagonal matrices, Duke Math. J. 51(4):981-996 (1984)

[76] E. Tsukerman and L. Williams, Bruhat interval polytopes, Adv. Math. 285: 766-810 (2015)

[77] C. Tomei, The Toda lattice, old and new, Journal of Geometric Mechanics 5(4):511-530 (2013)

Department of Mathematics, Ohio State University, Columbus, OH 43210

E-mail address: kodama@math.ohio-state.edu

Department of Mathematics, The University of Texas at Arlington, Arlington TX

E-mail address: bshipman@uta.edu 